\title{
The Dependence of Inspection-System Performance on Levels of Penalties and Inspection Resources
}

\author{
A. J. Goldman and M. H. Pearl \\ Institute for Basic Standards, National Bureau of Standards, Washington, D.C. 20234
}

(April 8, 1976)

\begin{abstract}
This paper presents three simple mathematical models, all of game-theoretic type, dealing with an inspector-inspectee relationship. The inspectee always tries to maximize his net gain, which is the amount he obtains by "cheating" less the amount he is penalized when caught. The first model assumes a zero-sum payoff and so the inspector tries to minimize the inspectee's net gain. In the second model, the inspector tries to deter cheating without concern for the extraction of penalties. In the third model we assume that the probabilistic pattern of the inspector's strategy is known to the inspectee and that the inspector constructs his strategy with this in mind. Each of these models is analyzed and optimal solutions are obtained. Several simple examples are presented to show the relation between the level of cheating and the levels of inspection resources and penalty.
\end{abstract}

Key words: Inspection; mathematical model; regulation; strategy; theory of games.

\section{Introduction}

This paper contains the formulation and analysis of three simple mathematical models, of gametheoretic type, oriented toward certain important aspects of the inspector-inspectee relationship. These models were initially designed in the course of a study performed for the NBS Office of Weights and Measures, while seeking techniques that might be useful to state "W\&M" agencies in employing their resources to achieve best protection of consumers against loss due to malfunctioning weighing and measuring devices used in retail trade. However, they also appear relevant to many other situations involving an inspector-inspectee relationship in which it is meaningful to assign numerical values to the detection and to the nondetection of malfunction.

For example, food packaging plants (the inspectees) are required to assure through self-policing that no more than a specified level of foreign matter (in the form either of less expensive substitutes or of "dirt") enters into the food they package. An agency of government (the inspector) is charged with guaranteeing to the public that these levels are not exceeded. If we can assign numerical values to (1) the benefit accruing to the packager (for example, in reduced operating expenses) for permitting systematic violation of the maximal level of foreign matter, and, (2) the penalty for being found in violation, then the models and analysis of this paper might well be pertinent. Similar comments can be made with "food purity" replaced by "consumer product safety."

The type of model at which we aim may also prove applicable to situations not customarily described in terms of inspectors and inspectees. For example, a department store (the inspector) wishes to combat thievery among its workforce (the inspectees) and establishes an internal security section with a fixed amount of resources. If numerical values can be specified for successful and for unsuccessful stealing, then models like those of this paper might be used to minimize the department store's loss. Many other scenarios can be formulated for which such models could describe "optimal strategies" for both the inspector and the inspectee:

Although the sort of analysis initiated below promises to have broad scope, it should be stated at the outset that the present models are not detailed and highly realistic ones, capable of giving "practical answers." Rather, they are intended both as preliminary explorations of how certain 
issues might be framed for quantitative analysis, and as concrete targets for criticisms and suggestions leading to superior versions.

The models ignore many features of the inspection process, for the sake of focusing sharply and clearly on certain other features. Some of the ignored aspects will be explicitly identified later, but for general perspective it should be noted that our emphasis here is one-sidedly on the "consumer protection" or "deterrence of cheating" element in inspection operations, as distinguished from the "help the inspectee (merchant) keep his device accurate" theme.

The features of the situation on which the models are intended to focus are the following:

(a) A commercial establishment, containing one or more measuring devices which "meters" its transactions with customers, has an economic incentive to "cheat" in connection with that device. The ugly word "cheat" is used as a pithy abbreviation for any of (i) deliberately causing the device to malfunction in the direction economically advantageous to the establishment (for example, "short weighing"), or (ii) knowingly permitting such a malfunction (originating through natural causes) to go uncorrected, or (iii) employing such a malfunctioning device, which though not explicitly recognized as such, was not properly checked for malfunction. Different establishments, with different dollarflows of transactions, will experience economic incentives of different magnitudes. The models contain a set of parameters $\left(V_{i}\right)$ representing these magnitudes.

(b) The inspection agency typically has only a limited quantity of "inspection resources" at its disposal, a quantity likely to be too small for frequent coverage of all the devices in its jurisdiction. The models contain parameters, $m$ and $n$, representing respectively, the inspection resources and the number of devices over which they must be spread.

(c) The detection of cheating leads to imposition of a "penalty" on the malefactor. This penalty might be the sum of (i) a monetary fine, (ii) the dollar-equivalent of interruption of the normal flow of business, and (iii) the economic loss due to diminished patronage following public disclosure of the cheating. The models include a parameter $(P)$ representing the size of this penalty.

(d) Our purpose in this paper is to begin the development of additional methodological tools for designing more effective and efficient responses by society to the existence of cheating. Those responses include both a punitive element (whose intensity is measured by $P$ ) and a "policing" element (whose intensity is represented by $m$ ). By determining how the level of illicit activity depends on $P$ and $m$, we hope to contribute to better understanding of the effectiveness of the twoelement response. By exhibiting the tradeoffs between $P$ and $m$ in the above dependence, we hope to contribute to better understanding of the efficiency with which that response is allocated between the two elements, though full achievement of that goal will also require models of the "cost" (as well as the "performance output") associated with various combinations of $P$ and $m$. These particular objectives, which of course remain relevant in a broad context of regulatory and general criminaljustice activity, have guided the development of the models to be described.

The mathematical formulation of a first model is given in the next section (sec. 2), and is accompanied by a discussion of some of that model's shortcomings in order to indicate directions for further research. The results from analyzing this Model 1 are presented in section 3, along with two simple illustrations; the analysis itself appears in section 4 (which the reader may prefer to omit). This model makes the "zero-sum" assumption-found in most game-theoretic literature-that the inspection agency's objectives are antithetical to those of the "inspectee," which implies in particular that the latter's loss through penalty-payment is the former's gain.

Section 5, in contrast, takes up a second model which is of nonzero-sum type; it treats the basic aim of the inspection activity as deterrence of cheating, with no separate value for the extraction of penalties after its detection. The "solution" of this Model 2 is presented and illustrated, with the supporting analysis deferred to section 6 .

In section 7 we drop the usual game-theoretic assumption that the players select their strategies simultaneously (or, at least, that the strategy chosen by each player does not depend on the strategy chosen by his opponent). Since the inspection procedure is an ongoing one, it would be expected that the inspectee, sooner or later, would be able to discern the probabilistic pattern of the 
inspector's choices. Thus, in Model 3 we assume that when the inspectee formulates his strategy he knows what the inspector's strategy will be. Moreover the inspector is aware of this and so constructs his strategy with it in mind. The solution of Model 3 was suggested by NBS colleague L.S. Joel; the results for Model 3 are presented in section 7 while section 8 supplies some of the longer derivations justifying these results.

\section{Formulation of Model 1}

This mathematical model takes the form of a 2-player zero-sum game. The "players" are the inspector (an aggregate representing the inspection agency) and the inspectee (an aggregate representing the establishments in which the measuring devices are used). There is some loss in reality through regarding these establishments as forming a single player with a single interest, but for the present model this potential turns out not to be realized.

The inspectee can either cheat, or not, for each of a set of devices $D_{1}, D_{2}, \ldots D_{n}$. The inspector selects devices for inspection, up to the limit of his resources. Detection of a cheat, if the device is inspected, is assumed certain. The data of the model are:

$n=$ number of devices,

$V_{i}=$ payoff to inspectee from cheating on $D_{i}$,

$P=$ penalty against inspectee for each detection of cheating,

$m=$ number of devices the inspector can examine $(m<n)$.

A strategy for the inspectee is an $n$-component vector

$$
\mathbf{c}=\left(c_{1}, c_{2}, \ldots, c_{n}\right)
$$

in which

$$
\begin{array}{ll}
c_{i}=1 & \text { if there is cheating on } D_{i}, \\
c_{i}=0 & \text { if there is no cheating on } D_{i} .
\end{array}
$$

The consideration of inspectee mixed strategies (probabilistic mixtures of ordinary strategies) is not necessary for the present model, i.e., the model has solutions which do not involve them, but we will also present those solutions which do include mixed inspectee strategies.

A strategy for the inspector is a specification of a subset $M$ of the set $N=\{1,2, \ldots, n\}$ such that $M$ has exactly $m$ members (notation: $|M|=m$ ). The interpretation is that $i \epsilon M$ if and only if $D_{i}$ is inspected. It turns out that consideration of mixed strategies for the inspector is required in order to solve the present model. Initially we define such a mixed strategy to be a vector $\mathbf{p}$, with a component $p(M)$ for each subset $M$ of $N$ such that $|M|=m$, and the properties that

$$
p(M) \geq 0, \sum_{M} p(M)=1 .
$$

The interpretation is given by

$$
p(M)=\operatorname{Prob}\left[\left\{D_{i}: i \epsilon M\right\} \text { are the devices inspected }\right] .
$$

With each such $\mathbf{p}$ can be associated the quantities

$$
\begin{aligned}
p_{i} & =\operatorname{Prob}\left[D_{i} \text { is inspected }\right] \\
& =\sum\{p(M): M \text { contains } i\} .
\end{aligned}
$$

Clearly each $p_{i}$ satisfies $0 \leq p_{i} \leq 1$. As will be proved in section 4 , the relation $\sum_{1}^{n} p_{i}=m$ holds. 
Conversely, consider any $n$-component vector $\left(p_{1}, p_{2}, \ldots, p_{n}\right)$ with

$$
0 \leq p_{i} \leq 1, \sum_{1}^{n} p_{i}=m
$$

As will also be proved in section 4 , there is then a mixed strategy $\mathbf{p}$ whose components $p(M)$ satisfy

$$
p_{i}=\sum\{p(M): M \text { contains } i\}
$$

for all $i \in N$. Thus we can and will regard a mixed strategy as a vector $\mathbf{p}=\left(p_{1}, p_{2}, \ldots, p_{n}\right)$ whose components $p_{i}$, interpreted as in (2.2), satisfy (2.3).

The net expected payoff to the inspectee, from device $D_{i}$, is the expected payoff from cheating minus the expected penalty, i.e.

$$
V_{i} c_{i}-P\left(c_{i} p_{i}\right)=\left[V_{i}-P p_{i}\right] c_{i} .
$$

Thus the total net expected payoff to the inspectee, if the two players choose respective mixed strategies $\mathbf{c}$ and $\mathbf{p}$, is

$$
F(\mathbf{c}, \mathbf{p})=\sum_{1}^{n}\left(V_{i}-P p_{i}\right) c_{i} .
$$

From the "zero-sum" assumption that the interests of the two players are diametrically opposed, it follows that $-F(\mathbf{c}, \mathbf{p})$ is the expected payoff to the inspector.

Before proceeding (in the next section) to present the "solution" given by this model-i.e., the "optimal strategies" for both players-we note some of the model's limitations and imperfections:

(a) The zero-sum assumption of diametrically opposed interests is not quite right unless one thinks of society (whose agent is the inspector) as seeking vengeance rather than deterrence. A second game-theoretic model, intended to give better expression to the "deterrence" theme, is formulated and analyzed in section 5 .

(b) The "cheat or no-cheat" dichotomy is clearly a severe idealization, ignoring as it does the possibility of introducing different degrees of bias into the devices (different amounts of cheating). To consider this dimension of the situation would also require formulating some mathematical representation of how the probability of detection depends on the degree of cheating, and could raise interesting questions concerning the role of the establishments' customers in performing part of the detection function (that of recognizing gross cheating). For the present we decline to explore these lines of generalization.

(c) Even with the above restriction, it seems odd to assume that cheating will be detected with certainty if the offending device is but inspected. This for example seems to rule out use of the model to examine the relative merits of training, or selecting, or otherwise encouraging inspectors to work more rapidly (in effect, increasing $m$ ), versus stressing the thoroughness or quality of their work (in effect, increasing he probability of cheat-detection). Fortunately, this limitation is only formal; a detection probability $\delta$ can be represented in the model simply by replacing the fixed penalty $P$, in (2.4), by the average penalty $P^{\prime}=P \delta$.

(d) A quite natural extension of the model would be the replacement of $P$, in (2.4), by devicespecific penalties $P_{i}$. (For example, the presence of site-dependent detection probabilities would lead, as above, to the use of $P \delta_{i}$ in place of $P$. Also, interruption of business or loss of patronage could be more serious at sites with a greater volume of transactions, suggesting the association of larger $P_{i}^{\prime}$ s with larger $V_{i}$ 's.) This would raise no real problem were we content with solving the model numerically, but it does interfere with achieving the kind of nearly closed-form solution presented in the next section, and it definitely contradicts the desire to maintain in the model a simple clear-cut scalar quantity representing the intensity of society's sanction against cheating. So, this complication will be omitted from the present paper. 
(e) If a serious level of cheating is detected, the inspector (government) may react with measures which are onerous to all inspectees (merchants), even those with properly functioning devices. Similarly, if detected cheating is publicized, honest as well as dishonest establishments may suffer from loss of public confidence. These considerations suggest an inadequacy in the way (2.4) associates penalty specifically to those devices at which cheating is discovered. Perhaps there should be a penalty $P^{*}$ which is "activated" if cheating is discovered at any device (or at more than some "threshold" number of devices). This possibility is also left for future investigation; its inclusion would preclude expressing the payoff function in terms of the $n \quad p_{i}$ 's rather than the much more numerous $p(M)$ 's.

(f) The inspector must decide which subset of the $n$ devices he will inspect, and the family of subsets which represent "allowable" outcomes of this decision are limited by the amount of inspection resources available. In the present model, that limitation is expressed by specifying the number $(m)$ of inspections which can be undertaken. This is clearly an idealization of the real-world situation in which some inspections may (predictably) require more time than others, some inspection sites are remote from the majority so that visiting them substantially reduces the number of other devices which can be inspected during a given time period, etc. One would like to improve the model by incorporating a more realistic representation of the family of "allowable" subsets from which the inspector can choose.

The preceding list suggests a number of directions for further analysis. But Model 1 and the others in this paper, despite their evident deficiencies, are felt to represent a suitable first step in focusing on the issues of interest.

\section{Results for Model 1}

In describing the results for the model formulated above, it is convenient to maintain the notation $N=\{1,2, \ldots, n\}$, and also to employ the notations

$$
T=\left\{i: V_{i}>P\right\}, \bar{T}=N-T=\left\{i: \quad V_{i} \leq P\right\} .
$$

The letter "T" was chosen because the devices $\left\{D_{i}: \quad i \in T\right\}$ are especially tempting choices for cheating.

The solution takes different forms according as

$$
m<|T|+\sum\left\{V_{i} / P: i \epsilon \bar{T}\right\} \quad(\text { Case I) }
$$

or its opposite

$$
m \geq|T|+\sum\left\{V_{i} / P: i \epsilon \bar{T}\right\} \quad(\text { Case II) }
$$

holds. If the $V_{i}$ are thought of as fixed, then this division into cases can be construed as partitioning the first quadrant of $(P, m)$-space into two regions. The Case I region corresponds (as will be seen) to situations where the inspectee's optimal strategy is to cheat on all devices, and so this region may be interpreted as representing grossly inadequate societal responses to the threat of cheating.

For each of the two cases, we will present a strategy $\mathbf{c}^{0}$ for the inspectee, a mixed strategy $\mathbf{p}^{0}$ for the inspector, and a number $F^{\mathrm{o}}$, such that

$$
\begin{array}{ll}
F\left(\mathbf{c}^{0}, \mathbf{p}\right) \geq F^{\mathbf{0}} & \text { for all } \mathbf{p}, \\
F\left(\mathbf{c}, \mathbf{p}^{0}\right) \leq F^{\mathbf{0}} & \text { for all } \mathbf{c} .
\end{array}
$$


These relations of course imply that

$$
F\left(\mathbf{c}^{\mathbf{0}}, \mathbf{p}^{\mathbf{0}}\right)=F^{\mathbf{0}} .
$$

Inequality (3.4) says that if the inspectee chooses strategy $\mathbf{c}^{0}$ then his payoff will be at least $F^{0}$ no matter what strategy the inspector picks, while (3.5) says that if the inspector chooses strategy $\mathbf{p}^{0}$ then the inspectee's payoff (the negative of his own) will be at most $F^{0}$ no matter what strategy the inspectee picks. On this basis, $\mathbf{c}^{\mathbf{0}}$ and $\mathbf{p}^{\mathbf{0}}$ are optimal strategies for the two players, and $F^{\mathbf{0}}$ is the value of the game (to the inspectee); we take $\mathbf{F}^{0}=\mathbf{F}\left(\mathbf{c}^{0}, \mathbf{p}^{0}\right)$ as the measure of average illicit net gains "predicted" by the model. This is the customary solution concept for zero-sum two-player games.

[A technical note to avoid a possible source of confusion: it can be proven from (3.4) and (3.5) that

$$
F^{o}=\max _{\mathbf{c}} \min _{\mathbf{p}} F(\mathbf{c}, \mathbf{p})=\min _{\mathbf{p}} \max _{\mathbf{c}} F(\mathbf{c}, \mathbf{p}) .
$$

Thus the value can be defined without reference to any particular $\mathbf{c}^{\mathbf{0}}$ and $\mathbf{p}^{\mathbf{0}}$. With this done, condition (3.4) defines the notion of an optimal strategy $\mathbf{c}^{0}$ without reference to any $\mathbf{p}^{0}$, while (3.5) defines the notion of an optimal strategy $\mathbf{p}^{0}$ without reference to any $\mathbf{c}^{0}$. Such definitions are in general possible only for zero-sum two-player games.]

For Case I, as already stated, the optimal strategy for the inspectee is to cheat on all devices; formally, $\mathbf{c}^{0}$ is given by

$$
c_{i}^{0}=1 \quad \text { for all } i \text {. }
$$

Any mixed strategy $\mathbf{p}^{0}$ for the inspector is optimal if it satisfies the "no overkill" condition

$$
P p_{i}^{0} \leq V_{i} \quad \text { for all } i \in \bar{T} \text {. }
$$

The value is given by

$$
F^{\mathbf{0}}=\sum_{1}^{\mathrm{n}} V_{i}-P m
$$

In Case II, it is convenient to partition $\bar{T}$ as $E \cup U$, where

$$
E=\left\{i: \quad V_{i}=P\right\}, \quad U=\left\{i: \quad V_{i}<P\right\}
$$

the symbols were chosen as the first letters of "equal" and "untempting." If strict inequality holds in (3.3), then the optimal strategies for the inspectee involve cheating on the tempting devices, but not on the untempting ones, i.e.,

$$
\begin{array}{ll}
c_{i}=1 & \text { for } i \in T, \\
c_{i}=0 & \text { for } i \in U, \\
c_{\mathrm{i}} \text { unspecified } & \text { for } i \in E .
\end{array}
$$

The phrasing of (3.13) was chosen to be compatible with the admission of mixed strategies for the inspectee (i.e., " $0 \leq c_{i} \leq 1$ " rather than " $c_{i}=0$ or 1 "). In the special case that equality holds in (3.3), there is an additional family of optimal strategies obtained by choosing a number $c$ with $0<c$ $\leq 1$ and (with (3.11) retained) replacing (3.12-3.13) with 


$$
\begin{array}{ll}
\mathrm{c}_{i}=c & \text { for } i \in U, \\
c_{i} \geq c & \text { for } i \in E .
\end{array}
$$

The optimal strategies for the inspector require always inspecting the tempting devices and avoiding "underkill" on the others, i.e.,

$$
p_{i}^{0}=1 \quad \text { for } i \epsilon T, \quad P p_{i}^{0} \geq V_{i} \quad \text { for } i \epsilon \bar{T}
$$

which implies $p_{i}^{0}=1$ for $i \epsilon E$. The value (whether (3.12-3.13) or $\left(3.12^{\prime}-3.13^{\prime}\right)$ is used) is

$$
F^{\mathbf{0}}=\sum\left\{V_{i}: i \in T\right\}-P|T| \text {. }
$$

Example 1: Equal-Sized Firms. This title is a somewhat free translation of the scenario-defining assumption that all $V_{i}$ have a common value $V$. Then (3.2) and (3.9) give

$$
F^{0}=n V-P m \quad \text { if } m<|T|+(V / P)\{n-|T|\},
$$

while (3.3) and (3.15) give

$$
F^{0}=(V-P)|T| \quad \text { if } m \geq|T|+(V / P)\{n-|T|\} .
$$

If $P<V$ then $|T|=n$, so that (since we assume $m<n$ ) the defining condition of (3.16) holds and hence (3.16) rather than (3.17) is the case. If $V \leq P$ then $|T|=0$, so that either (3.16) or (3.17) holds depending on whether or not $P<n V / m$. Our main interest is not in the optimal strategies but rather in the dependence of the "net illicit gain" $F^{0}$, a measure of (imperfect) performance by the inspection system, as a function of $P$ and $m$. This dependence is summarized in figure 1 , in which $F^{0}$ is continuous across the curve (an equilateral hyperbola) separating the two regions shown. The notation $V_{\text {tot }}=\sum_{1}^{n} V_{i}(=n V)$ is used.

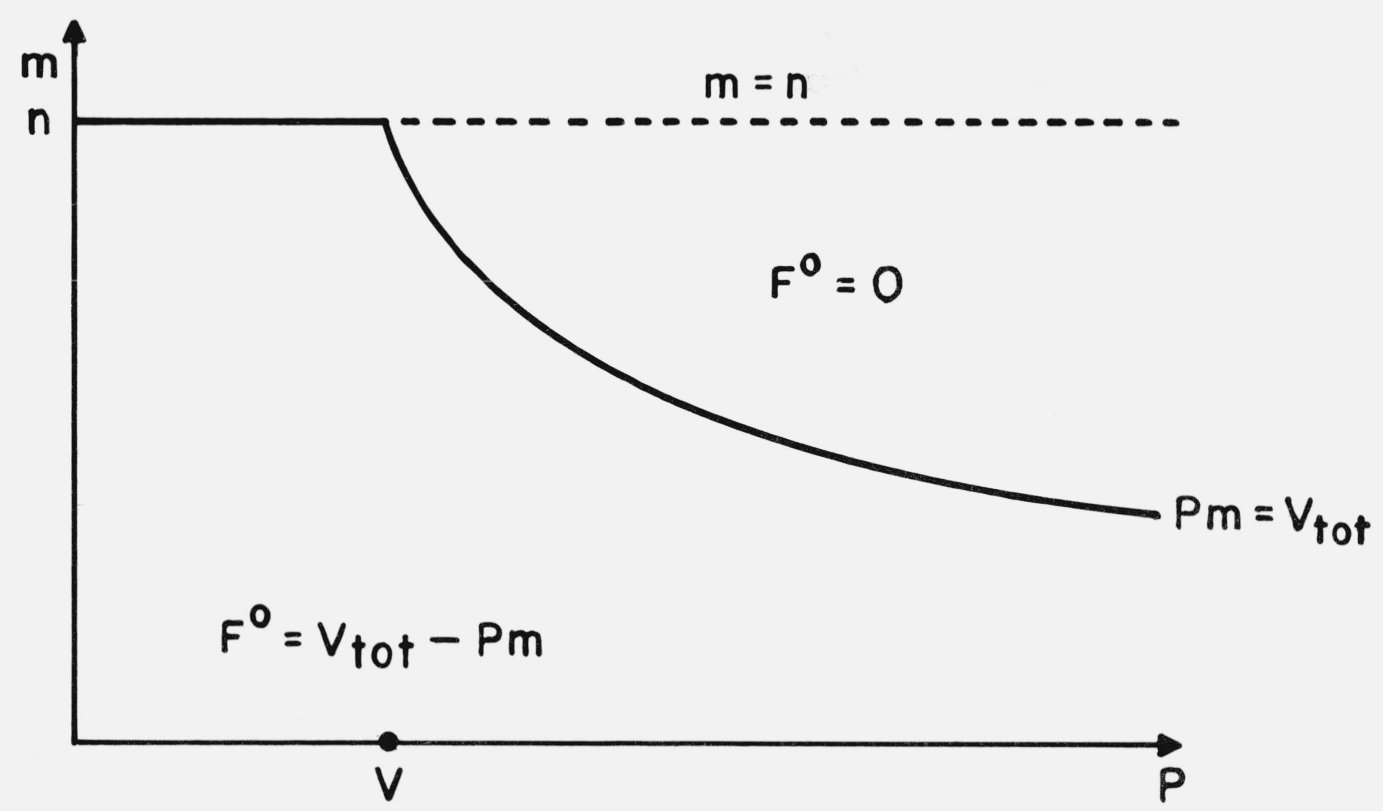

Figure 1. Net illicit gain (equal-sized firms). 
Example 2: Big Firms, Small Firms. Here there are $n_{b}$ "big firms" each with $V_{i}=V_{b}$, and $n_{s}$ "small firms" each with $V_{i}=V_{s}$, where $V_{b}>V_{s}$. We have $V_{\text {tot }}=n_{b} V_{b}+n_{s} V_{s}$. If $P<V_{s}$, so that $|T|$ $=n$ and $\bar{T}$ is empty, then (3.2) and (3.9) give (since $m<n$ )

$$
F^{\mathrm{o}}=V_{\mathrm{tot}}-P m
$$

If $V_{s} \leq P<V_{b}$, so that $|T|=n_{b}$ and $|\bar{T}|=n_{s}$, then (3.2) and (3.9) give

$$
F^{\mathrm{o}}=V_{\mathrm{tot}}-P m \quad \text { if } m<n_{b}+\left(V_{s} / P\right) n_{s},
$$

while (3.3) and (3.15) give

$$
F^{\mathbf{0}}=\left(V_{b}-P\right) n_{b} \quad \text { if } m \geq n_{b}+\left(V_{s} / P\right) n_{s} .
$$

If $P>V_{b}$, so that $T$ is empty and $|\bar{T}|=n$, then (3.2) and (3.9) give

$$
F^{0}=V_{\mathrm{tot}}-P m \quad \text { if } m<V_{\mathrm{tot}} / P,
$$

while (3.3) and (3.15) give

$$
F^{0}=0 \quad \text { if } m \geq V_{\text {tot }} / P .
$$

These results are summarized in figure $2 . F^{0}$ is continuous across each of the three boundary curves. Note that the region corresponding to (3.18) is "inspection-saturated"; a stiffer penalty, rather than more inspection activity, is needed to reduce the inspectee's expected payoff $F^{0}$ from his optimal strategy (cheat at the big firms, but not at the small ones). (See table 1.)

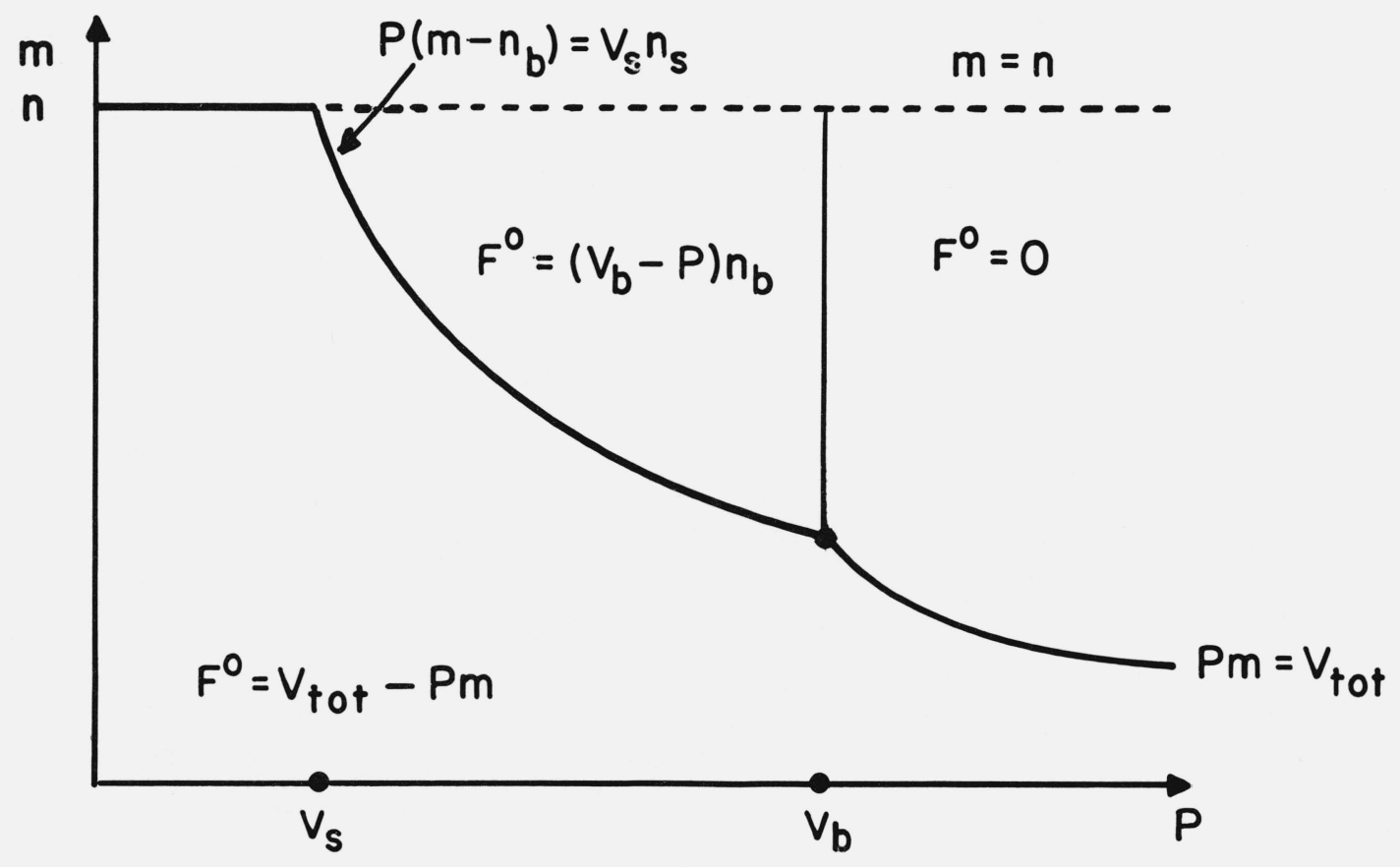

FigURE 2. Net illicit gain (big firms, small firms). 
TABLE 1. Results for Model I

\begin{tabular}{|c|c|c|c|c|}
\hline & Case Definition & Inspectee's Strategy & Inspector's Strategy & Payoff \\
\hline 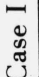 & $m<|T|+\sum\left\{V_{i} / P: \quad i \epsilon \bar{T}\right\}$ & $c_{i}^{0}=1$ for all $i$ & $\begin{array}{r}p_{i}^{o} \leq V_{i} / p \text { for } i \in \bar{T} \\
0 \leq p_{i}^{o} \leq 1 \text { for } i \in T\end{array}$ & $F^{0}=V_{\text {tot }}-P m$ \\
\hline 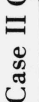 & $\boldsymbol{m} \geq|T|+\sum\left\{\boldsymbol{V}_{i} / P:\left.\quad i\right|_{\boldsymbol{\epsilon}} \bar{T}\right\}$ & $\begin{array}{c}c_{i}^{o}=1 \text { for } i \epsilon T \\
c_{i}^{o}=0 \text { for } i \epsilon U \\
0 \leq c_{i}^{o} \leq 1 \text { for } i \epsilon E\end{array}$ & $\begin{array}{l}p_{i}^{o}=1 \text { for } i \text { ge } T \\
p_{i}^{i} \geq V_{i} / P \text { for } i \in T\end{array}$ & $\begin{array}{c}F^{\mathbf{o}=} \geq\left\{V_{i}: i \epsilon T\right\} \\
-P|T|\end{array}$ \\
\hline
\end{tabular}

\section{Analysis for Model 1}

This section contains the mathematical justification of the results presented in section 3 . Readers preferring to do so can go directly to section 5 without loss of continuity.

A first technical point to be settled is the permissability of passing from the original definition of a mixed strategy for the inspector, namely that of a vector with components $p(M)$ satisfying $(2.1)$ where $M$ ranges over all subsets of $N$ with $|M|=m$-to the subsequent definition as a vector $\mathbf{p}$ with components $p_{i}$ satisfying (2.3). It will first be shown that each collection $\{p(M)\}$ obeying (2.1) leads via (2.2) to a set of $p_{i}$ obeying (2.3); then it will be proved that each such $\mathbf{p}=\left(p_{i}\right)$ arises from such a collection $\{p(M)\}$.

For the first purpose, consider any collection $\{p(M)\}$ obeying (2.1), and let $a_{i}=1$ for all $i \epsilon N$. With $p_{i}$ defined by (2.2), we clearly have $p_{i} \geq 0$ as well as

$$
p_{i}=\sum\{p(M): \quad M \text { with } i \epsilon M\} \leq \sum_{M} p(M)=1,
$$

and also

$$
\begin{aligned}
\sum_{1}^{n} p_{i} & =\sum_{i} a_{i} \sum\{p(M): \quad M \text { with } i \in M\} \\
& =\sum\left\{a_{i} p(M): \quad \text { pairs }(i, M) \text { with } i \epsilon M\right\} \\
& =\sum_{M} p(M) \sum\left\{a_{i}: \quad i \epsilon M\right\} \\
& \left.=\sum_{M} p(M) \cdot m \quad \text { (since } a_{i}=1 \text { and }|M|=m\right) \\
& =m,
\end{aligned}
$$

so that the $p_{i}$ obey (2.3).

For the second purpose define, for each subset $Q$ of $N$ for which $|Q|=m$, a vector $\pi_{Q}$ whose components are given by

$$
\pi_{Q}(M)=0 \quad \text { for } M \neq Q ; \quad \pi_{Q}(Q)=1 .
$$

Note that the $\pi_{Q}$ are the vertices of the set (hypercube) $S^{*}$ consisting of vectors $\pi$ with components $p(M)$ satisfying (2.1). Next, for each subset $Q$ of $N$ for which $|Q|=m$, define a vector $\mathbf{p}_{Q}$ with components given by

$$
p_{Q i}=1 \quad \text { if } i \epsilon Q ; p_{Q i}=0 \quad \text { if } i \in N-Q .
$$


The set $S$, of all vectors $\mathbf{p}$ with components $p_{i}$ satisfying (2.3), is the intersection of a hypercube with a hyperplane; its vertices must be the intersections of the hyperplane with the edges or vertices of the hypercube, from which it readily follows that the vertices of $S$ are precisely the vectors $\mathbf{p}_{Q}$.

Finally, for each $i \in N$ and each subset $M$ of $N$ with $|M|=m$, let $a_{i M}=1$ if $i \epsilon M$ and $a_{i M}=0$ otherwise. Then the relation (2.2) can be written

$$
p_{i}=\sum_{M} a_{i M} p(M)
$$

or, in linear transformation notation, as

$$
\mathbf{p}=A \boldsymbol{\pi}
$$

It is readily vertified that $\mathbf{p}_{Q}=A \pi_{Q}$. Now any vector $\mathbf{p} \epsilon S$ can be written as a convex combination of the vertices of $S$, i.e.,

$$
\mathbf{p}=\sum_{Q} \lambda_{Q} p_{Q} \quad\left(\lambda_{Q} \geq 0, \sum_{Q} \lambda_{Q}=1\right)
$$

The vector

$$
\pi=\sum_{Q} \lambda_{Q} \pi_{Q}
$$

as a convex combination of the vertices of $S^{*}$, is itself in $S^{*}$, and from the linearity of $A$ we have

$$
A \boldsymbol{\pi}=\sum_{Q} \lambda_{Q} A \pi_{Q}=\sum_{Q} \lambda_{Q} \mathbf{p}_{Q}=\mathbf{p} .
$$

This proves, as desired, that every $p \in S$ arises via (2.2) from some $\pi \epsilon S^{*}$.

To prove that the strategies presented in section 3 are indeed optimal, it is convenient to set

$$
v_{i}=V_{i} / P
$$

and to work, not with the payoff function $F$ of (2.4), but rather with

$$
f(\mathbf{c}, \mathbf{p})=F(\mathbf{c}, \mathbf{p}) / P=\sum_{1}^{n}\left(v_{i}-p_{i}\right) c_{i} .
$$

Note that

$$
T=\left\{i: \quad v_{i}>1\right\}, \quad \bar{T}=\left\{i: \quad v_{i} \leq 1\right\} .
$$

We turn first to Case I, which according to (3.2) is characterized by

$$
m<|T|+\sum\left\{v_{i}: \quad i \in \bar{T}\right\} .
$$

Using (3.9), we set

$$
f^{\circ}=F \%=\sum_{1}^{n} v_{i}-m \text {. }
$$

Following (3.7), let $\mathbf{c}^{0}$ be defined by $c_{i}^{\mathbf{0}}=1$ for all $i$. Then by (2.3) and (4.2)

$$
f\left(\mathbf{c}^{\mathbf{0}}, \mathbf{p}\right)=\sum_{1}^{n}\left(v_{\mathrm{i}}-p_{i}\right)=\sum_{1}^{n} v_{i}-m=f^{\mathbf{o}}
$$

for all mixed strategies $\mathbf{p}$ for the inspector. 
Next, consider any mixed strategy $\mathbf{p}^{\mathbf{0}}$ such that $p_{i}^{0} \leq v_{i}$ for all $i \in T$. Then, since $\mathbf{p}^{0} \leq 1<v_{i}$ for all $i \epsilon T$, it follows that for all $\mathbf{c}$,

$$
\begin{gathered}
f\left(\mathbf{c}, \mathbf{p}^{0}\right)=\sum_{1}^{n}\left(v_{i}-p_{i}^{0}\right) c_{\mathrm{i}} \\
\leq \sum_{1}^{n}\left(v_{i}-p_{i}^{0}\right)=f^{0} .
\end{gathered}
$$

Such mixed strategies $\mathbf{p}^{0}$ do exist, for if $m \leq \sum\left\{v_{i}\right.$ : $\left.i \epsilon \bar{T}\right\}$ they can be chosen with $p_{i}^{0}=0$ for all $i \in T$, while if

$$
\sum\left\{v_{i}: \quad i \epsilon \bar{T}\right\}<m<|T|+\sum\left\{v_{i}: \quad i \epsilon \bar{T}\right\}
$$

then (4.4) assures the existence of some with $p_{i}^{0}=v_{i}$ for all $i \epsilon \bar{T}$.

The preceding material shows that $\left(\mathbf{c}^{0}, \mathbf{p}^{0}, \mathbf{f}^{\circ}\right)$ give optimal strategies and the value for the game with payoff function $f$, so that $\left(\mathbf{c}^{\mathbf{0}}, \mathbf{p}^{\mathbf{0}}, F^{\mathbf{o}}\right)$ give the corresponding information for the original game. Moreover, the indicated optimal strategies are the only ones. To see this, note first that the strictness of the inequality in (4.4) permits a choice of $\mathbf{p}^{\mathbf{0}}$ in (4.6) for which $p_{i}^{0}<v_{i}$ for all $i \epsilon \bar{T}$, and then equality will hold in (4.6) only for $\mathbf{c}=\mathbf{c}^{\mathbf{0}}$. (A separate but easy argument is needed if $\bar{T}$ is empty.) Next, if $\mathbf{p}$ is any mixed strategy for which

$$
I_{1}=\left\{i: \quad p_{i}>v_{i}, i \in \bar{T}\right\}
$$

is nonempty, then define $\mathbf{c}$ by $c_{i}=0$ for $i \epsilon I_{1}$ and $c_{\mathrm{i}}=1$ for all $i \epsilon N-I_{1}$. One obtains

$$
\begin{aligned}
f(\mathbf{c}, \mathbf{p}) & =\sum\left\{v_{i}-p_{\mathrm{i}}: \quad i \in N-I_{1}\right\} \\
& =\sum_{1}^{n}\left(v_{i}-p_{i}\right)-\sum\left\{\left(v_{i}-p_{i}\right): \quad i \in I_{1}\right\} \\
& =f^{0}-\sum\left\{\left(v_{i}-p_{i}\right): \quad i \in I_{1}\right\}>f^{0},
\end{aligned}
$$

and the existence of a $\mathbf{c}$ with this property rules out the optimality of $\mathbf{p}$.

Next we present the analysis for Case II, which according to (3.3) is characterized by

$$
m \geq|T|+\sum\left\{v_{i}: \quad i \epsilon \bar{T}\right\}=|T \cup E|+\sum\left\{v_{i}: \quad i \epsilon U\right\} .
$$

Using (3.12), we put

$$
f^{o}=\sum\left\{v_{i}: \quad i \in T\right\}-|T|
$$

With $\mathbf{c}^{0}$ defined by (3.11-3.13),

$$
\begin{gathered}
f\left(\mathbf{c}^{\mathbf{0}}, \mathbf{p}\right)=\sum\left\{\left(v_{i}-p_{i}\right): \quad i \epsilon T\right\}+\sum\left\{\left(1-\mathrm{p}_{i}\right) c_{i}: \quad i \epsilon E\right\} \\
\geq \sum\left\{\left(v_{i}-1\right): \quad i \epsilon T\right\}=f^{0}
\end{gathered}
$$

for all $\mathbf{p}$. On the other hand, for $\mathbf{p}^{\mathbf{0}}$ satisfying (3.14), i.e.,

$$
p_{i}^{0}=l \quad \text { for } i \epsilon T ; p_{i}^{0} \geq v_{i} \quad \text { for } i \epsilon \bar{T},
$$

we have

$$
f\left(\mathbf{c}, \mathbf{p}^{0}\right) \leq \sum\left\{\left(v_{i}-1\right): \quad i \epsilon T\right\}=f^{0}
$$


for all $\mathbf{c}$. So $\left(\mathbf{c}^{\mathbf{0}}, \mathbf{p}^{\mathbf{0}}, \mathbf{f}^{\mathbf{0}}\right)$ form an optimal solution. The existence of mixed strategies $\mathbf{p}^{\mathbf{0}}$ obeying (4.10) is assured by (4.7).

Once again, there are no other optimal inspector strategies. To see this, note first that equality holds in (4.9) only if

$$
p_{i}=1 \quad \text { for all } i \in T \text {, }
$$

which will therefore be assumed in the balance of this paragraph. If

$$
I_{2}=\left\{i: \quad p_{i}<v_{i}, i \epsilon \bar{T}\right\}
$$

is nonempty then one can define $\mathbf{c}$ by

$$
c_{i}=1 \quad \text { for } i \epsilon T \cup I_{2}, \quad c_{i}=0 \quad \text { for } i \epsilon \bar{T}-I_{2} \text {, }
$$

yielding

$$
f(\mathbf{c}, \mathbf{p})=f^{0}+\sum\left\{\left(v_{i}-p_{i}\right): \quad i \in I_{2}\right\}>f^{o}
$$

which shows that $\mathbf{p}$ is not optimal.

Next we inquire whether there are other optimal inspectee strategies; mixed strategies $\left(0 \leq c_{i}\right.$ $\leq 1)$ are admitted in the discussion. Nite first that equality holds in (4.11) only if

$$
c_{i}=1 \quad \text { for all } i \in T \text {, }
$$

which is therefore assumed in the balance of this paragraph. Suppose that

$$
K=\left\{k: \quad c_{k}>0, \quad k \in U\right\}
$$

is nonempty; we will attempt to deduce from this that $\mathbf{c}$ is not optimal. If strict inequality holds in (4.7), one can choose $\mathbf{p}$ to satisfy (4.10) with $p_{k}>v_{k}$ for all $k \in K$; thus

$$
f(\mathbf{c}, \mathbf{p})=f^{0}+\sum\left\{\left(v_{k}-p_{k}\right) c_{k}: \quad k \in K\right\}<f^{0},
$$

so that $\mathbf{c}$ is not optimal. Now assume (4.7) holds with equality. If there exist distinct $k \epsilon K$ and $i \epsilon E$ $\cup U$ with $c_{i}<c_{k}$, then choose any positive $\delta$ with

$$
\delta<\min \left\{v_{i}, 1-v_{k}\right\}
$$

and define $\mathbf{p}$ by

$$
\begin{gathered}
p_{j}=1 \quad \text { for } j \in T \cup E-\{i\}, \\
p_{j}=v_{j} \quad \text { for } j \epsilon U-\{i, k\}, \\
p_{i}=v_{i}-\delta, p_{k}=v_{k}+\delta .
\end{gathered}
$$


Then $\mathbf{p}$ is a mixed strategy, and

$$
f(\mathbf{c}, \mathbf{p})=f^{\mathbf{o}}+\delta c_{i}+(-\delta) c_{k}<f^{0}
$$

showing that $\mathbf{c}$ is not optimal. The only remaining possibility is that equality holds in (4.7) and, for some $c$ with $0<c \leq 1$,

$$
c_{i}=c \quad \text { for } i \epsilon U ; c_{i} \geq c \quad \text { for } i \epsilon E .
$$

In this situation, for any $\mathbf{p}$,

$$
\begin{aligned}
f(\mathbf{c}, \mathbf{p})= & \sum\left\{\left(v_{i}-p_{i}\right): \quad i \in T\right\}+\sum\left\{\left(1-p_{i}\right) c_{\mathrm{i}}: \quad i \in E\right\} \\
& +c \sum\left\{\left(v_{\mathrm{i}}-p_{\mathrm{i}}\right): i \in U\right\} \\
= & f^{0}+\sum\left\{\left(1-p_{i}\right): i \in T\right\}+\sum\left\{\left(1-p_{i}\right) c_{i}: \quad i \epsilon E\right\} \\
& +c\left[\sum\left\{v_{i}: \quad i \epsilon U\right\}-\sum\left\{p_{i}: \quad i \in U\right\}\right] \\
\geq & f^{0}+\sum\left\{\left(1-p_{i}\right): \quad i \in T\right\}+c \sum\left\{\left(1-p_{i}\right): \quad i \in E\right\} \\
& +c\left[(m-|T \cup E|)-\left(m-\sum\left\{p_{i}: \quad i \in T \cup E\right\}\right)\right] \\
= & f^{0}+(1-c) \sum\left\{\left(1-p_{\mathrm{i}}\right): \quad i \epsilon T\right\} \geq f^{\circ},
\end{aligned}
$$

showing that $\mathbf{c}$ is indeed optimal.

One further technical question is whether the use of mixed strategies for the inspector is really necessary. Might not the inspector have "unmixed" (or "pure") strategies which are optimal? Such a strategy $\mathbf{p}^{0}$, besides obeying (2.3), would also satisfy

$$
p_{i}^{0}=0 \text { or } 1 \quad \text { for all } i \in N \text {. }
$$

In Case II, we see from (4.10) that this would require all $p_{i}^{0}=1$, so that (2.3) would be satisfied only in the uninteresting case $m=n$. For Case I, assuming no $v_{i}$ exactly equal to $1,(4.12)$ and the condition $p_{i}^{0} \leq v_{i}$ for all $i \in \bar{T}$ imply that $p_{i}^{0}=0$ for all $i \epsilon \bar{T}$, so that (2.3) can be satisfied if and only if $|T| \geq m$, a condition which does not follow from the defining characteristic (4.4) of Case I. So mixed strategies are indeed needed in Case II, and also in Case I when $|T|<m$.

\section{Formulation and Results for Model 2}

In what follows, the payoff function for the inspectee is as before in (2.4), namely

$$
F(\mathbf{c}, \mathbf{p})=\sum_{1}^{n}\left[V_{i}-P p_{i}\right] c_{i} .
$$

We now regard the inspector's aim, however, as that of minimizing the total loss (e.g., to consumers) as a result of cheating. The payoff function for the inspector is therefore taken initially to be

$$
G(\mathbf{c}, \mathbf{p})=-\sum_{1}^{n} V_{i} c_{i},
$$

rather than the previous $-F(\mathbf{c}, \mathbf{p})$. 
The solution concept typically employed for such nonzero-sum (and "noncooperative") games is that of an equilibrium point (EP), namely a pair $\left(\mathbf{c}^{\mathbf{0}}, \mathbf{p}^{\mathbf{0}}\right)$ of strategies such that

$$
\begin{aligned}
& F\left(\mathbf{c}, \mathbf{p}^{0}\right) \leq F\left(\mathbf{c}^{\mathbf{0}}, \mathbf{p}^{0}\right) \quad \text { for all } \mathbf{c}, \\
& G\left(\mathbf{c}^{\mathbf{0}}, \mathbf{p}\right) \leq G\left(\mathbf{c}^{\mathbf{0}}, \mathbf{p}^{0}\right) \quad \text { for all } \mathbf{p} .
\end{aligned}
$$

Such a pair has the "stability" property that if both players adopt them, then neither has incentive for a unilateral change in his strategy.

This approach is not helpful in the present situation, since any strategy $\mathbf{p}^{\mathbf{0}}$ for the inspector is part of some EP, $\left(\mathbf{c}^{\mathbf{0}}, \mathbf{p}^{\mathbf{0}}\right)$. To show this, one need merely choose $\mathbf{c}^{\mathbf{0}}$ to maximize $F\left(\mathbf{c}, \mathbf{p}^{\mathbf{0}}\right)$; then (5.3) is obeyed, and since $G(\mathbf{c}, \mathbf{p})$ depends only on its first argument, (5.4) is also satisfied. Thus, the formulation provides no guidance for the inspector.

Two methods for escaping this unsatisfactory state of affairs have been considered. One of them, which involves abandonment of a strictly game-theoretic approach, is discussed in sections 7 and 8. The other ("Model 2"), to be treated in this section and the next one, introduces a slightly different objective for the inspector, namely to minimize the total loss due to undetected cheating. Thus (5.2) is replaced by

$$
G(\mathbf{c}, \mathbf{p})=-\sum_{1}^{n} \mathrm{~V}_{i} c_{i}\left(1-p_{i}\right) .
$$

A rationale for this objective-reasonable, but in the writers' opinion less than compelling-can be given in terms of a scenario in which society's response to cheating is so structured that the penalty for a second offense is prohibitive to the inspectee. (Thus, $P$ now represents the penalty for a first (detected) offense.) For such a scenario, it is plausible that future cheating would occur precisely at the sites of current undetected cheating, so that its extent is measured by (5.5).

We now proceed to describe the equilibrium points $\left(\mathbf{c}^{\mathbf{0}}, \mathbf{p}^{\mathbf{0}}\right)$ of the game with payoff functions (5.1) and (5.5); the analysis supporting these results is given in section 6. The associated payoff values, $F^{0}=F\left(\mathbf{c}^{0}, \mathbf{p}^{0}\right)$ and $G^{0}=G\left(\mathbf{c}^{0}, \mathbf{p}^{0}\right)$, will also be presented.

The devices may be assumed numbered so that

$$
V_{1} \geq V_{2} \geq \ldots \geq V_{n}
$$

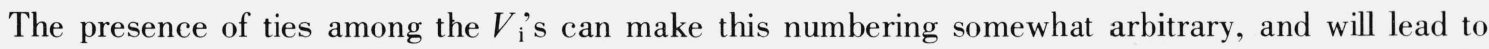
some complications (technical rather than substantive) in describing the solution of the model. In addition to the sets

$$
T=\left\{i: V_{i}>P\right\}, \quad \bar{T}=\left\{i: V_{i} \leq P\right\}
$$

defined earlier, it is convenient to define the sets

$$
\begin{aligned}
& N_{m}=\{1,2, \ldots, m\}, \quad N_{m}^{0}=\left\{i: \quad V_{i}=V_{m}\right\} \\
& N_{m}^{+}=\left\{i: \quad V_{i}>V_{m}\right\}, \quad N_{m}^{-}=\left\{i: \quad V_{i}<V_{m}\right\} .
\end{aligned}
$$

The three sets $N_{m}^{+}, N_{m}^{-}$and $N_{m}^{0}$ partition $N$; either or both of the first two might be empty, but $N_{m}^{0}$ contains at least the member $m$.

The discussion of Model 2, like that of Model 1, splits into cases. First assume

$$
V_{m}>P \quad(\text { Case I). }
$$


As in Case I of Model 1, all equilibrium points $\left(\mathbf{c}^{\mathbf{0}}, \mathbf{p}^{0}\right)$ for this case involve cheating on all devices, i.e.,

$$
c_{i}^{\mathbf{0}}=1 \quad \text { for all } i .
$$

The strategies $\mathbf{p}^{0}$ involve always inspecting those devices more tempting than the $m$ th, and never inspecting those less tempting, i.e.,

$$
\begin{gathered}
p_{i}^{0}=1 \quad \text { for } i \epsilon N_{m}^{+}, \quad p_{i}^{0}=0 \quad \text { for } i \epsilon N_{m}^{-}, \\
0<p_{i}<1 \quad \text { for } i \epsilon N_{m}^{0}, \sum\left\{p_{i}^{0}: i \epsilon N_{m}^{0}\right\}=m-\left|N_{m}^{+}\right| .
\end{gathered}
$$

Substituting (5.9-5.11) into (5.1) and (5.5), we find that the inspectee's payoff is

$$
F^{\mathbf{0}}=\sum_{1}^{n} V_{i}-P m
$$

the same as for Case I of Model 1, and similarly the inspector's payoff is

$$
G^{0}=-\left[V_{m}\left\{n-\left|N_{m}^{-}\right|-m\right\}+\sum\left\{V_{i}: \quad i \epsilon N_{m}^{-}\right\}\right] .
$$

Next, assume

$$
V_{m}=P \quad \text { (Case II) }
$$

There are two subcases. If $m$ is not the last member of $N_{m}^{o}$, i.e.,

$$
\left|N_{m}^{+}\right|+\left|N_{m}^{\mathbf{o}}\right|>m, \quad(\text { Case IIA })
$$

then the equilibrium points and payoff functions are given by the same formulas (5.9-5.13) as for Case I. But if

$$
\left|N_{m}^{+}\right|+\left|N_{m}^{\mathbf{0}}\right|=m, \quad \text { (Case IIB) }
$$

then the equilibrium points require always cheating on both the tempting and untempting devices, e.g.,

$$
\begin{array}{ll}
c_{i}^{\mathbf{0}}=1 & \text { for } i \in N_{m}^{+}=T, \\
c_{i}^{\mathbf{0}}=1 & \text { for } i \epsilon N_{m}^{-}=U,
\end{array}
$$

together with a "floor" under the frequency of cheating on the other devices:

$$
c_{i}^{0} \geq V_{m+1} / P \quad \text { for } i \in N_{m}^{0}=E .
$$

The inspection policies are given by

$$
\begin{array}{ll}
p_{i}^{0}=1 & \text { for } i \epsilon N_{m}^{+} \cup N_{m}^{0}=T \cup E, \\
p_{i}^{0}=0 & \text { for } i \epsilon N_{m}^{-}=U .
\end{array}
$$


The corresponding values of the payoff functions are

$$
F^{\mathbf{0}}=\sum_{1}^{n} V_{i}-P m
$$

as before, and

$$
G^{\mathbf{o}}=-\sum\left\{V_{i}: \quad i \in N_{m}^{-}\right\}
$$

which in view of (5.16) "matches" with (5.13).

The remaining case is

$$
V_{m}<P \quad \text { (Case III). }
$$

If the condition

$$
m \geq|T \cup E|+\sum\left\{V_{i} / P: \quad i \epsilon U\right\} \quad \text { (Case IIIA) }
$$

holds, there is a family of equilibrium points given by

$$
\begin{array}{ll}
c_{i}^{\mathbf{0}}=1 & \text { for } i \in T, \\
0 \leq c_{i}^{\mathbf{0}} \leq 1 & \text { for } i \in E, \\
c_{i}^{\mathbf{0}}=0 & \text { for } i \in U, \\
p_{i}^{\mathbf{0}}=1 & \text { for } i \in T \cup E \\
p_{i}^{\mathbf{0}} \geq V_{i} / P & \text { for } i \in U .
\end{array}
$$

The associated payoff values are

$$
\begin{aligned}
F^{\mathbf{0}} & =\sum\left\{V_{i}: \quad i \in T\right\}-P|T|, \\
G^{\mathbf{0}} & =0 .
\end{aligned}
$$

A second and more complicated type of equilibrium point exists when

$$
m \leq|T \cup E|+\sum\left\{V_{i} / P: \quad i \in U\right\} . \quad \text { (Case IIIB). }
$$

For its description, let

$$
L=\left\{i: \quad V_{m}<V_{i}<P\right\}
$$

so that $N_{m}^{+}$is partitioned as $T \cup E \cup L$ and $U$ as $\mathrm{L} \cup N_{m}^{\mathbf{o}} \cup N_{m}^{-}$. Furthermore, divide $N_{m}^{-}$into blocks; a "block" is defined to be a maximal succession of integers $i$ with a common $V_{i}$-value. Let $B_{b}$ denote the $b$ th block, and $V_{b}^{*}$ its associated $V_{i}$-value; the blocks are numbered $B_{1}$ through $B_{\beta}$ in descending order of $V_{b}^{*}$. It will also be convenient to regard $N_{m}^{0}$ as an initial block $B_{0}$, so that $V_{0}^{*}=$ $V_{m}$. Because $T \cup E \cup L$ does not contain m,

$$
\Delta=m-|T \cup E|-\sum\left\{V_{i} / P: \quad i \epsilon L\right\}>0 .
$$

Choose the smallest integer $\alpha$ for which

$$
\Delta \leq \sum_{b=0}^{\alpha}\left|B_{b}\right| V_{b}^{*} / P
$$


by (5.33), such an $\alpha$ must exist. Also, let

$$
\lambda=\Delta-\sum_{b=0}^{\alpha-1}\left|B_{b}\right| V_{b}^{*} / P,
$$

so that

$$
0<\lambda \leq\left|B_{\alpha}\right| V_{\alpha}^{*} / P
$$

Next, choose $c_{m}^{\mathbf{0}}$ so that

$$
\begin{aligned}
c_{m}^{0}=V_{\alpha}^{*} / V_{m} & \text { if } \lambda<\left|B_{\alpha}\right| V_{\alpha}^{*} / P, \\
V_{\alpha+1}^{*} / V_{m}<c_{m}^{0} \leq V_{\alpha}^{*} / V_{m} & \text { if } \lambda=\left|B_{\alpha}\right| V_{\alpha}^{*} / \mathrm{P},
\end{aligned}
$$

where in (5.39) we set $V_{\alpha+1}^{*}=0$ if $\alpha=\beta$. Then an equilibrium point $\left(\mathbf{c}^{0}, \mathbf{p}^{\boldsymbol{0}}\right)$ is given by

$$
\begin{array}{ll}
c_{i}^{\mathbf{0}}=1 & \text { for } i \epsilon T, \\
c_{i}^{0} \geq c_{m}^{0} V_{m} / P & \text { for } i \epsilon E, \\
c_{i}^{\mathbf{0}}=c_{m}^{0} V_{m} / V_{i} & \text { for } i \epsilon L, \\
c_{i}^{\mathbf{0}}=c_{m}^{0} V_{m} / V_{i} & \text { for } \begin{cases}i \epsilon B_{b} & \text { with } 0 \leq b<\alpha \\
i \epsilon B_{\alpha} & \text { if } \lambda=\left|B_{\alpha}\right| V_{\alpha}^{*} / P,\end{cases} \\
c_{i}^{\mathbf{0}}=1 & \text { for } \begin{cases}i \epsilon B_{\alpha} & \text { if } \lambda<\left|B_{\alpha}\right| V_{\alpha}^{*} / P \\
i \epsilon B_{b} & \text { with } \alpha<b \leq \beta,\end{cases} \\
p_{i}^{\mathbf{0}}=1 & \text { for } i \epsilon T \cup E, \\
p_{i}^{\mathbf{0}}=V_{i} / P & \text { for } i \epsilon L, \\
p_{i}^{\mathbf{0}}=V_{i} / P & \text { for } \begin{cases}i \epsilon B_{b} \quad \text { with } 0 \leq b<\alpha \\
i \epsilon B_{\alpha} & \text { if } \lambda=\left|B_{\alpha}\right| V_{\alpha}^{*} / P,\end{cases} \\
p_{i}^{\mathbf{0}} \leq V_{i} / P & \text { for } i \epsilon B_{\alpha} \quad \text { if } \lambda<\left|B_{\alpha}\right| V_{\alpha}^{*} / P, \\
p_{i}^{0}=0 & \text { for } i \epsilon B_{b} \quad \text { with } \alpha<b \leq \beta, \\
\sum\left\{p_{i}^{0}: \quad i \epsilon B_{\alpha}\right\}=\lambda . &
\end{array}
$$

The associated payoffs are given by

$$
\begin{aligned}
& F^{\mathbf{0}}=\sum\left\{V_{i}: \quad i \epsilon T\right\}-P|T|+\sum_{b \geq \alpha}\left|B_{b}\right| V_{b}^{*}-P \lambda=V_{\mathrm{tot}}-P m, \\
& \mathrm{G}^{\mathbf{0}}=-\left[\left\{\sum_{b>\alpha}\left|B_{b}\right| V_{b}^{*}+\left\{n-m-\sum_{b>\alpha}\left|B_{b}\right|\right\} c_{m}^{0} V_{m}\right\}\right] .
\end{aligned}
$$

Note that in (5.52), the factor $c_{m}^{0} \mathrm{~V}_{m}$ in the last summand is equal to $V_{\alpha}^{*}$ if $\lambda<\left|B_{\alpha}\right| V_{\alpha}^{*} / P$. But if

$$
\lambda=\left|B_{\alpha}\right| V_{\alpha}^{*} / P
$$


then $\mathrm{c}_{m}^{0} \mathrm{~V}_{m}$ has as range of variation the interval $\left(V_{\alpha+1}^{*}, V_{\alpha}^{*}\right)$ determined by (5.39), so that $G^{0}$ as given by (5.52) has a corresponding range of variation. This illustrates the general theoretical possibility, in a nonzero sum game, for different equilibrium points (i.e., "solutions") to have different associated payoff levels. The present model exhibits this phenomenon only for the particular "coincidence" described by (5.53).

It should also be observed that Cases IIIA and IIIB may coexist, giving rise to different equilibrium points. This occurs precisely when

$$
m=|T \cup E|+\sum\left\{V_{i} / P: \quad i \in U\right\},
$$

which in the context of Case IIIB implies that

$$
\alpha=\beta, \quad \lambda=\left|B_{\beta}\right| V_{n} / P,
$$

so that the above-mentioned nonuniqueness of $G^{\circ}$ occurs.

The general results presented above will now be illustrated using the same two special scenarios employed for Model 1, namely the "equal-sized firms" case (all $V_{i}=V$ ) and the "big firms, small firms" scenario $\left(V_{i}=V_{b}\right.$ for $1 \leq i \leq n_{b}, V_{i}=V_{s}$ for $\left.n_{b}<i \leq n_{b}+n_{s}=n, V_{b}>V_{s}\right)$.

ExAmple 1: Equal-Sized Firms. Here $\left|N_{m}^{+} \cup N_{m}^{-}\right|=0$ and $\left|N_{m}^{0}\right|=n$. Since $n>m$, comparison with (5.16) shows that Case IIB is ruled out. If $V \geq P$, so that Case I or Case IIA holds, then (5.12) and (5.13) give

$$
F^{\mathbf{o}}=V_{\mathrm{tot}}-P m, G^{\mathbf{0}}=-V(n-m) \quad(\text { if } V \geq P) .
$$

Now suppose Case III is in effect, i.e., $V<P$. Then $|T \cup E \cup L|=0$. By reference to (5.25), (5.31) and (5.32), Case IIIA gives rise to

$$
\left.F^{\mathbf{o}}=G^{\mathbf{o}}=0 \quad \text { (if } V<P \text { and } P m \geq V_{\text {tot }}\right) .
$$

For Case IIIB, one has

$$
\alpha=\beta=0, \quad \Delta=\lambda=m, \quad\left|B_{\alpha}\right| \mathrm{V}_{\alpha}^{*} / P=V_{\mathrm{tot}} / P
$$

so that (5.51) and (5.52), together with (5.38) and (5.39), yield

$$
\begin{array}{ll}
F^{0}=V_{\mathrm{tot}}-P m & \left(\text { if } V<P \text { and } P m \leq V_{\mathrm{tot}}\right), \\
G^{0}=-V(n-m) & \text { (if } \left.V<P \text { and } P m<V_{\mathrm{tot}}\right), \\
-V(n-m) \leq G^{0} \leq 0 & \text { (if } \left.V<P \text { and } P m=V_{\mathrm{tot}}\right) .
\end{array}
$$

The dependence of $G^{0}$ on $P$ and $m$ is summarized in figure 3 ; the dependence of $F^{0}$ is given by the earlier figure 1. Note, in figure 3 , that $G^{0}$ is not continuous across the hyperbola $P m=V_{\text {tot }}$; as shown by (5.60), it is not single-valued on this curve, and its range of variation at each point of the curve is precisely the interval between its limits as the curve is approached from within each of the two regions identified in the figure. In the lower region, $G^{0}$ does not depend on $P$; this can be interpreted as a situation of "penalty saturation," in which stiffer penalties yield no improvement unless accompanied by greater inspection resources.

ExAmple 2: Big Firms, Little Firms. The situation here is more complicated. First suppose $V_{b}$ $>V_{s} \geq P$. Then either Case I or Case II applies, so that by (5.12) 


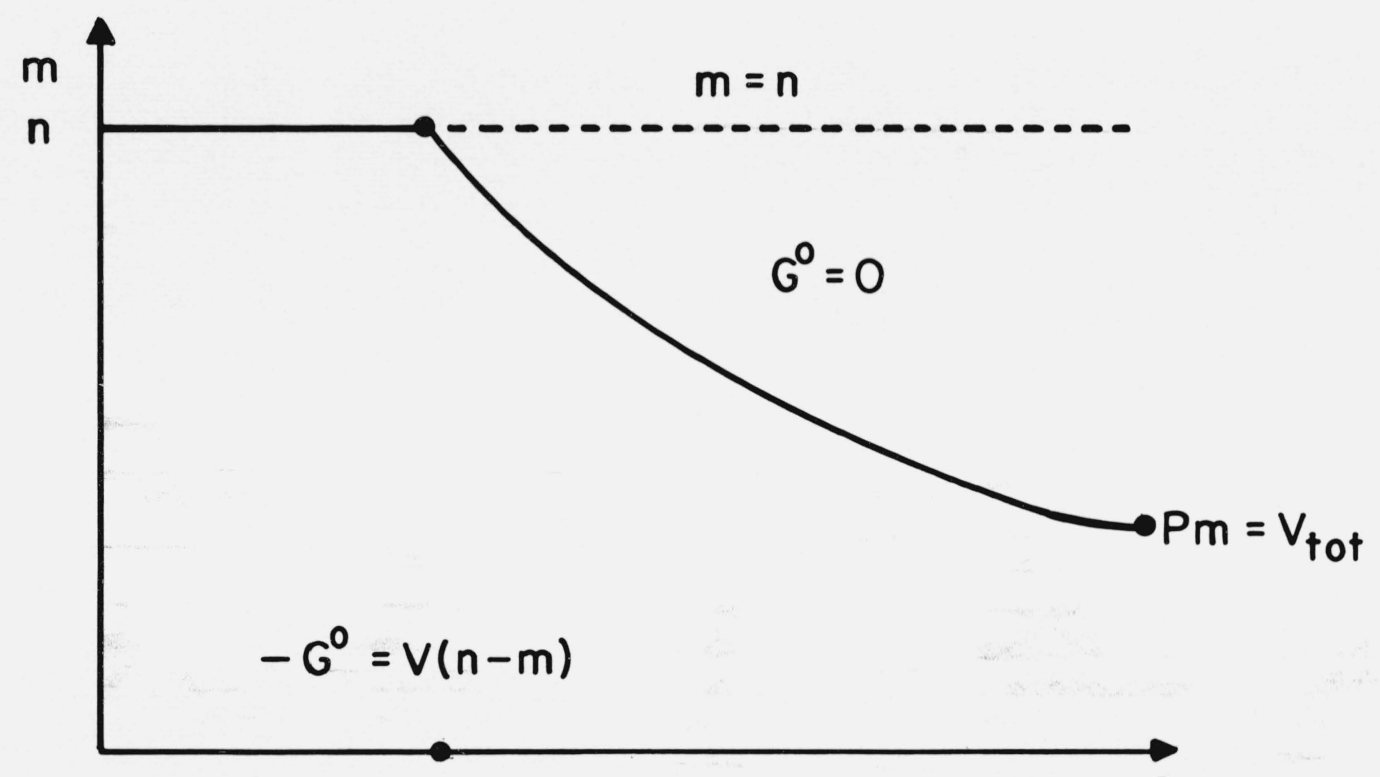

FIGURE 3. Undetected illicit activity (equal-sized firms).

$$
F^{\mathrm{o}}=V_{\mathrm{tot}}-P m \quad\left(\text { if } V_{b}>V_{s} \geq P\right)
$$

If $m \leq n_{b}$, then $V_{m}=V_{b},\left|N_{m}^{o}\right|=n_{b}$ and $\left|N_{m}^{-}\right|=n_{s}$; Case I applies, and (5.13) yields

$$
G^{\mathbf{o}}=-\left(V_{\mathrm{tot}}-V_{b} m\right) \quad\left(\text { if } \quad V_{b}>V_{s} \geq P \text { and } m \leq n_{b}\right) .
$$

If $m>n_{b}$, then $V_{m}=V_{s},\left|N_{m}^{+}\right|=n_{b}$, and $\left|N_{m}^{o}\right|=n_{s}$; Case IIB is ruled out, and (5.13) yields

$$
\mathrm{G}^{0}=-\mathrm{V}_{s}(\mathrm{n}-\mathrm{m}) \quad\left(\text { if } V_{b}>V_{s} \geq P \text { and } m>n_{b}\right) .
$$

Next, suppose $V_{b} \geq P>V_{s}$. If $m \leq n_{b}$, then Case I or Case II holds, with $V_{m}=V_{b}\left|N_{m}^{0}\right|=$ $\mathrm{n}_{b}$ and $\left|N_{b}\right|=n_{s}$; formulas (5.61) and (5.62) also apply to this situation. If $m>n_{b}$, then $V_{m}=V_{s}$ and Case III holds, with $|T \cup E|=n_{b},|L|=0$ and $|U|=\left|N_{m}^{0}\right|=n_{s}$. Case IIIA is characterized by $P\left(m-n_{b}\right) \geq V_{s} n_{s}$, in which case (5.31) and (5.32) yield

$$
F^{\mathbf{o}}=\left(V_{b}-P\right) n_{b}, \quad G^{\mathbf{o}}=0 \quad(\text { Case IIIA }) .
$$

Case IIIB is characterized in the present context by $P\left(m-n_{b}\right) \leq V_{s} n_{s}$; it has $\alpha=\beta=0, \Delta=\lambda=$ $m-n_{b}$ and $\left|B_{\alpha}\right| V_{\alpha}^{*} / P=V_{s} n_{s} / P$, so that (5.5l) yields

$$
F^{\mathbf{o}}=V_{\mathrm{tot}}-P m \quad(\text { Case IIIB) }
$$

and (5.52) yields

$$
G^{0}=-V_{s}(n-m) \quad \text { if } P\left(m-n_{b}\right)<V_{s} n_{s} \quad \text { (Case IIIB), }
$$




$$
-V_{s}(n-m) \leq G^{0} \leq 0 \quad \text { if } P\left(m-n_{b}\right)=V_{s} n_{s} \quad \text { (Case IIIB) }
$$

Finally, suppose $P>V_{b}>V_{s}$, so that Case III applies with $|T \cup E|=0$. If $m \leq n_{b}$, then $|L|=$ $0,\left|N_{m}^{\mathbf{0}}\right|=n_{b}, \beta=1$ and $\left|B_{1}\right|=n_{s}$. Case IIIA is characterized by $P m \geq V_{\text {tot }}$, and by (5.31)-(5.32) it yields $F^{0}=G^{0}=0$. Case IIIB is characterized by $P m \leq V_{\text {tot }}$, and has $\Delta=m$. There are two subcases. First, if $P m \leq V_{b} n_{b}$ then $\alpha=0, \lambda=m$, and (5.51)-(5.52) yield $F^{\mathbf{o}}=V_{\text {tot }}-P m$ together with

$$
\begin{array}{lr}
G^{0}=-\left(V_{\mathrm{tot}}-V_{b} m\right) & \text { (if } \left.P m<V_{b} n_{b}\right), \\
-\left(V_{\mathrm{tot}}-V_{b} m\right) \leq G^{\mathbf{0}} \leq-V_{s}(n-m) & \text { (if } \left.P m=V_{b} n_{b}\right) .
\end{array}
$$

Second, if $V_{b} n_{v}<P m \leq V_{\mathrm{tot}}$, then $\alpha=1, \lambda=m-V_{b} n_{b} / P$, and (5.51)-(5.52) yield $F^{0}=V_{\mathrm{tot}}-P m$ together with

$$
\begin{array}{ll}
G^{\mathbf{0}}=-V_{s}(n-m) & \left(\text { if } P m<V_{\mathrm{tot}}\right), \\
-V_{s}(n-m) \leq G^{\mathbf{0}} \leq 0 & \text { (if } \left.P m=V_{\mathrm{tot}}\right) .
\end{array}
$$

Still supposing $P>V_{b}>V_{s}$, now assume $m>n_{b}$. Then $|L|=n_{b}\left|N_{m}^{0}\right|=n_{s}$ and $\alpha=\beta=0$. Case IIIA is characterized by $P m \geq V_{\text {tot }}$, and as above yields $F^{\mathbf{o}}=G^{\mathbf{0}}=0$. Case IIIB is characterized by $P m \leq V_{\text {tot }}$, has $\Delta=\lambda=m-V_{b} n_{b} / P$; (5.51)-(5.52) yield $F^{0}=V_{\text {tot }}-P m$ together with

$$
\begin{array}{ll}
G^{\mathbf{0}}=-V_{s}(n-m) & \left(\text { if } P m<V_{\mathrm{tot}}\right), \\
-V_{s}(n-m) \leq G^{\mathbf{0}} \leq 0 & \left(\text { if } P m=V_{\mathrm{tot}}\right) .
\end{array}
$$

The dependence of $F^{0}$ upon $P$ and $m$ is the same as in Model 1 , portrayed in the earlier figure 2 . The dependence of $G^{0}$ is summarized in figure $4 . G^{0}$ is continuous on the horizontal boundary segment (of the line $m=n_{b}$ ), but is neither continuous across nor single-valued along the three hyperbolic boundary arcs. Note the presence of two distinct regions exhibiting penalty-saturation. (See table 2.) 
TABLE 2. Results for Model 2

\begin{tabular}{|c|c|c|c|c|}
\hline & Case Definition & Inspectee's Strategy & Inspector's Strategy & Payoffs \\
\hline 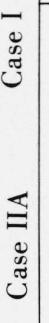 & $\begin{array}{c}V_{m}=P \\
\left|N_{m}^{+}\right|+\left|N_{m}^{0}\right|>m\end{array}$ & $\begin{array}{l}c_{i}^{0}=1 \text { for all } i \\
c_{i}^{\mathbf{0}}=1 \text { for all } i\end{array}$ & $\begin{array}{l}p_{i}^{0}=1 \text { for } i \epsilon N_{m}^{+} \\
p_{i}^{o}=0 \text { for } i \epsilon N_{m}^{-} \\
0<p_{i}^{0}<1 \text { for } i \epsilon N_{m}^{0} \\
\sum\left\{p_{i}^{0}: \quad \text { i } \epsilon \mathrm{N}_{m}^{o}\right\}=m-\left|N_{m}^{+}\right| \\
p_{i}^{o}=1 \text { for } i \epsilon N_{m}^{+} \\
p_{i}^{o}=0 \text { for } i \epsilon N_{m}^{-} \\
0<p_{i}^{0}<1 \text { for } i \epsilon N_{m}^{o} \\
\sum\left\{p_{i}^{o}: \quad \text { i } \epsilon \mathrm{N}_{m}^{o}\right\}=\mathrm{m}-\left|\mathrm{N}_{m}^{+}\right|\end{array}$ & $\begin{array}{c}F^{0}=V_{\text {tot }}-P m \\
-G^{0}=\left[V_{m}\left\{n-\left|N_{m}^{-}\right|-M\right\}+\right. \\
\left.\sum\left\{V_{i}: \text { i } \in N_{m}^{-}\right\}\right] \\
F^{\mathbf{0}}=V_{\mathrm{tot}}-P m \\
-G^{\mathbf{0}}=\left[V_{m}\left\{n-\left|N_{m}^{-}\right|-m\right\}+\right. \\
\left.\sum\left\{V_{i}: \quad i \in N_{m}^{-}\right\}\right]\end{array}$ \\
\hline 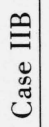 & $\begin{array}{l}V_{m}=P \\
\quad\left|N_{m}^{+}\right|+\left|N_{m}^{0}\right|=m\end{array}$ & $\begin{array}{l}c_{i}^{0}=1 \text { for } i \epsilon N^{+}{ }_{m}=T \\
c_{i}^{0}=1 \text { for } i \epsilon N^{-}{ }_{m}=U \\
c_{i}^{0} \geq V_{m+1} / P \text { for } i \epsilon N_{m}^{0}=E\end{array}$ & $\begin{array}{l}p_{i}^{0}=1 \text { for } i \epsilon N_{m}^{+} \cup N_{m}^{0}=T \cup E \\
p_{i}^{0}=0 \text { for } i \epsilon N_{m}^{-}=U\end{array}$ & $\begin{array}{c}F^{\mathrm{o}}=V_{\mathrm{tot}}-P m \\
\left.-G^{\mathbf{0}}=\sum\left\{V_{i}: \quad i \in N_{m}^{-}\right\}\right]\end{array}$ \\
\hline 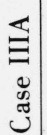 & $\begin{array}{l}\quad V_{m}<P \\
m \geq|T \cup E|+\leq\left\{V_{i} / P:\right. \\
i \in U\}\end{array}$ & $\begin{array}{l}c_{i}^{\mathbf{0}}=1 \text { for } i \epsilon T \\
0 \leq c_{i}^{0} \leq, 1 \text { for } i \epsilon E \\
c_{i}^{0}=0 \text { for } i \epsilon U\end{array}$ & $\begin{array}{l}p_{i}^{0}=1 \text { for } i \epsilon T \cup E \\
p_{i}^{0} \geq V_{i} / P \text { for } i \epsilon U\end{array}$ & $\begin{array}{c}F^{\mathbf{0}}=\sum\left\{V_{\mathrm{i}}: \quad i \in T\right\}-P|T| \\
G^{\mathbf{0}}=0\end{array}$ \\
\hline 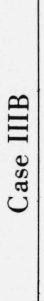 & $\begin{array}{l}\quad V_{m}<P \\
m \leq|T \cup E|+\mathfrak{Z}\left\{V_{i} / P:\right. \\
i \in U\}\end{array}$ & $\begin{array}{c}c_{i}^{0}=1 \text { for } i \epsilon T \\
c_{i}^{0} \geq c_{m}^{0} V_{m} / P \text { for } i \epsilon E \\
c_{i}^{0}=c_{m}^{0} V_{m} / P \text { for } i \epsilon L \\
c_{i}^{0}=c_{m}^{0} V_{m} / V_{i} \text { for }\left\{\begin{array}{l}i \epsilon B_{b} \text { with } 0 \leq b<\alpha \\
i \epsilon B_{\alpha} \text { if } \lambda=\left|B_{\alpha}\right| V_{\alpha}^{*} / P\end{array}\right. \\
c_{\mathrm{i}}^{0}=1 \text { for }\left\{\begin{array}{l}i \epsilon B_{\alpha} \text { if } \lambda<\mid B_{\alpha} V_{\alpha}^{*} / P \\
i \epsilon B \text { with } \alpha<b \leq \beta\end{array}\right.\end{array}$ & $\begin{array}{c}p_{i}^{0}=1 \text { for } i \epsilon T \cup E \\
p_{i}^{0}=V_{i} / P \text { for } i \in L \\
p_{i}^{0}=V_{i} / P \text { for }\left\{\begin{array}{l}i \epsilon B_{b} \text { with } 0 \leq b<\alpha \\
i \epsilon B_{\alpha} \text { if } \lambda=\left|B_{\alpha}\right| V_{\alpha}^{*} / P\end{array}\right. \\
p_{i}^{0} \leq V_{i} / P \text { for } i \epsilon B_{\alpha} \text { if } \lambda<\left|B_{\alpha}\right| V_{\alpha}^{*} / P \\
p_{i}^{0}=0 \text { for } i \epsilon B_{\alpha} \text { with } \alpha<b \leq \beta\end{array}$ & $\begin{array}{c}F^{\mathbf{0}}=V_{\mathrm{tot}}-P m \\
-G^{0}=\sum_{b>\alpha}\left|B_{\alpha}\right| V_{b}^{*}+ \\
\left.\left\{n-\sum_{b>\alpha} \mid B_{\alpha}\right\}\right\} c_{m}^{0} V_{m}\end{array}$ \\
\hline
\end{tabular}




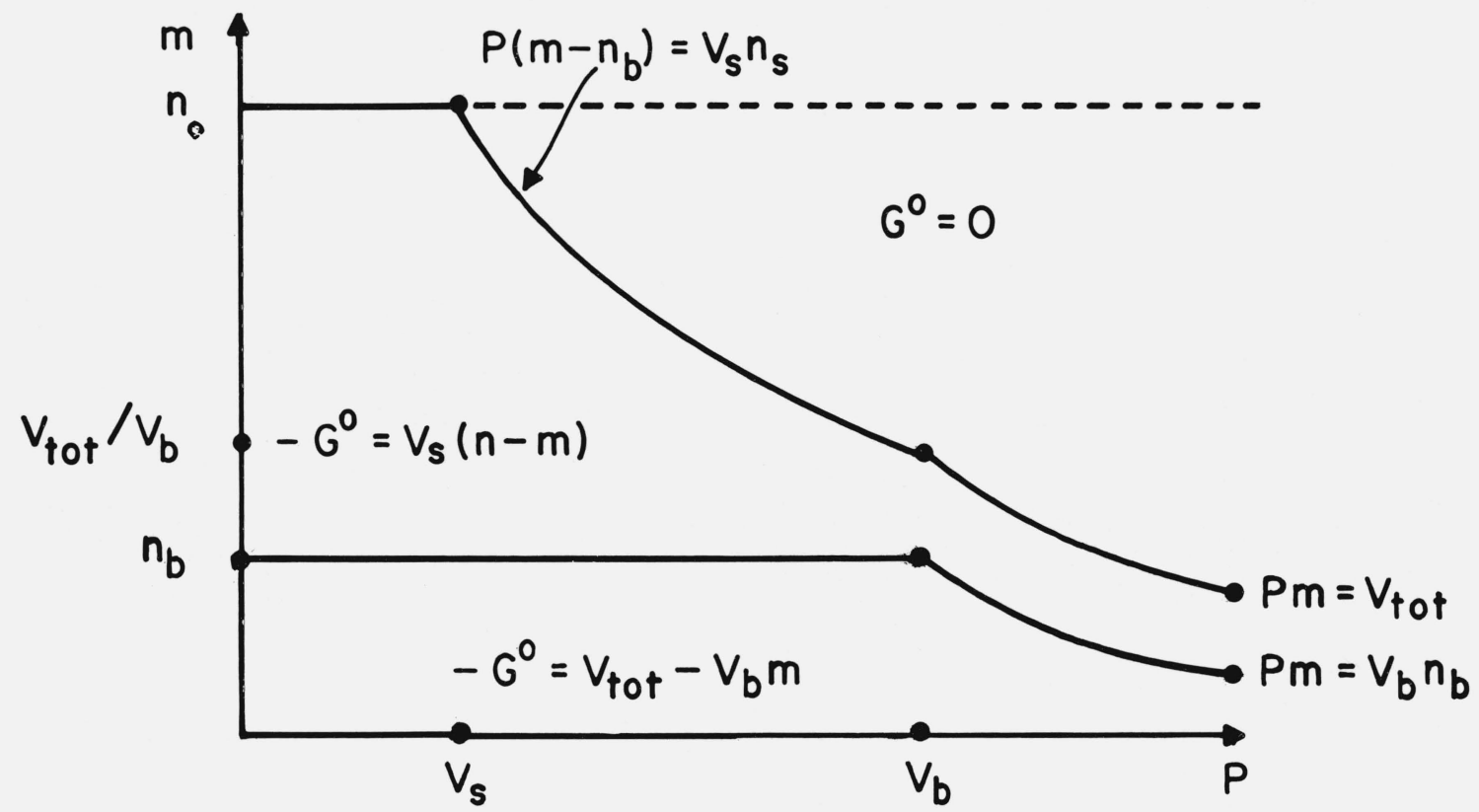

FIGURE 4. Undetected illicit activity (big firms, small firms).

\section{Analysis for Model 2}

The aim in this section is to prove the results cited in section 5, i.e., to determine the equilibrium points $\left(\mathbf{c}^{0}, \mathbf{p}^{\mathbf{0}}\right)$ of the game with payoff functions

$$
F(\mathbf{c}, \mathbf{p})=\sum_{1}^{n}\left[V_{i}-P p_{i}\right] c_{i}, \quad G(\mathbf{c}, \mathbf{p})=-\sum_{1}^{n} V_{i} c_{i}\left(1-p_{i}\right) .
$$

Mixed strategies for the inspectee (i.e., $0 \leq c_{i} \leq 1$ ) are permitted. The convention

$$
V_{1} \geq V_{2} \geq \ldots \geq V_{n}
$$

will remain in force, as will the definitions given in section 5 of the sets $T, \bar{T}, E, U, N_{m}$, $N_{m}^{+}, N_{m}^{\circ}$, and $N_{m}^{-}$and $L$. Of the two conditions defining an equilibrium point, (5.3) is equivalent to the pair of requirements

$$
\begin{array}{ll}
c_{\mathrm{i}}^{\mathbf{0}}=1 & \text { if } V_{i}>P p_{i}^{\mathbf{0}}, \\
\mathrm{c}_{\mathrm{i}}^{\mathbf{0}}=0 & \text { if } V_{i}<P p_{i}^{\mathbf{0}},
\end{array}
$$

while satisfying (5.4) is equivalent to choosing $\mathbf{p}^{\mathbf{0}}$ so as to achieve 


$$
\max _{\mathbf{p}} \sum_{1}^{n}\left(V_{i} c_{i}^{0}\right) p_{i} \quad \text { subject to (2.3). }
$$

An immediate consequence of (6.3) is that

$$
c_{i}^{0}=1 \quad \text { for } i \epsilon T .
$$

Another preliminary observation is that

$$
\left|N_{m}^{+}\right|+\left|N_{m}^{\mathbf{0}}\right| \geq m
$$

since $N_{m}^{+} \cup N_{m}^{\mathbf{o}}$ contains $N_{m}$.

We begin with the situation

$$
V_{m}>P \quad \text { (Case I). }
$$

Here $N_{m}^{+} \cup N_{m}^{0}$ is a subset of $T$, so that (6.6) yields

$$
c_{i}^{0}=1 \quad \text { for } i \epsilon N_{m}^{+} \cup N_{m}^{\mathbf{o}},
$$

and since $N_{m}^{+}$contains the $\left|N_{m}^{+}\right|$largest $V_{i}$ 's and $\left|N_{m}^{+}\right|<m$, it follows from (6.5) that

$$
p_{i}^{0}=1 \quad \text { for } i \epsilon N_{m}^{+} .
$$

Combining (6.7), (6.9) and (6.5), it follows that

$$
\begin{gathered}
\sum\left\{p_{i}^{0}: \quad i \epsilon N_{m}^{0}\right\}=m-\left|N_{m}^{+}\right| \\
p_{i}^{0}=0 \quad \text { for } i \in N_{m}^{-} .
\end{gathered}
$$

By (6.3) and (6.12),

$$
c_{i}^{\mathbf{0}}=1 \quad \text { for } i \epsilon N_{m}^{-}
$$

Conversely, any $\left(\mathbf{c}^{\mathbf{0}}, \mathbf{p}^{\mathbf{0}}\right)$ which satisfies (6.9-6.13) also satisfies (6.3) through (6.5), and so is an equilibrium point. This completes the analysis of Case I.

Next assume

$$
V_{m}=P \quad \text { (Case II), }
$$

so that $N_{m}^{+}, N_{m}^{\mathrm{o}}, N_{m}^{-}$coincide with $T, E, U$ respectively. By (6.6),

$$
c_{i}^{0}=1 \quad \text { for } i \in N_{m}^{+},
$$

so that (6.5) implies

$$
p_{i}^{0}=1 \quad \text { for } i \epsilon N_{m}^{+} \text {. }
$$


Define the set

$$
N_{m 1}^{\mathbf{o}}=\left\{i: \quad i \in N_{m}^{\mathbf{o}}, c_{i}^{\mathbf{o}}=1\right\}
$$

we note that for $i \epsilon N_{m}^{0}-N_{m 1}^{0}$, (6.3) implies $0 \geq P-P p_{i}^{\circ}$, i.e.,

$$
p_{i}^{0}=1 \quad \text { for } i \epsilon N_{m}^{0}-N_{m 1}^{0} .
$$

First assume that ${ }^{1}$

$$
\left|N_{m}^{+}\right|+\left|N_{m 1}^{0}\right|>m \quad(\text { Case IIA })
$$

Then (6.5) implies

$$
\begin{array}{ll}
p_{i}^{\mathbf{o}}=0 & \text { for } i \in N_{m}^{\mathrm{o}}-N_{m 1}^{\mathrm{o}}, \\
p_{i}^{\mathbf{0}}=0 & \text { for } i \epsilon N_{m}^{-} .
\end{array}
$$

Comparison of (6.18) and (6.20) shows that $\mathrm{N}_{m}^{o}-N_{m 1}^{o}$ is empty, i.e.,

$$
c_{i}^{0}=1 \quad \text { for } i \in N_{m}^{0},
$$

while (6.21) and (6.3) imply

$$
c_{i}^{0}=1 \quad \text { for } i \in N_{m}^{-},
$$

and (6.16) and (6.20) imply

$$
\sum\left\{p_{i}^{o}: \quad i \in N_{\mathrm{m}}^{\mathrm{o}}\right\}=m-\left|N_{m}^{+}\right| .
$$

Conversely, such a $\left(\mathbf{c}^{0}, \mathbf{p}^{\text {o) }}\right.$, which has the same structure as in Case I, satisfies (6.3-6.5) and so is an equilibrium point. The defining condition (6.19) takes the form

$$
\left|N_{m}^{+}\right|+\left|N_{m}^{0}\right|>m
$$

i.e., $m$ is not the last member of $N_{m}^{0}$.

Next assume that

$$
\left|N_{m}^{+}\right|+\left|N_{m 1}^{o}\right| \leq m \quad \text { (Case IIB) }
$$

Then (6.5) together with (6.18) imply

$$
p_{i}^{0}=1 \quad \text { for } i \in N_{m}^{0} .
$$

From this and (6.16) it follows that

$$
m=\sum_{1}^{n} p_{i}^{0} \geq\left|N_{m}^{+}\right|+\left|N_{m}^{0}\right|,
$$

and comparison with (6.7) shows that

$$
\left|N_{m}^{+}\right|+\left|N_{m}^{o}\right|=m
$$

${ }^{1}$ The analysis which follows will show that this kind of correspondence coincides with Case IIA as defined in section 5 (5.15). The same situation will recur in later subcases. 
(hence there is no overlap with Case IIA's range) and that

$$
p_{i}^{0}=0 \quad \text { for } i \in N_{m}^{-} \text {. }
$$

By (6.29) and (6.3)

$$
c_{i}^{0}=1 \quad \text { for } i \in N_{m}^{-} .
$$

From (6.5) it follows, in view of (6.27) and (6.29), that

$$
V_{i} c_{i} \geq V_{j} c_{j} \quad \text { for } i \epsilon N_{m}^{0} \text { and } j \in N_{m}^{-},
$$

which by (6.28) and (6.30) is equivalent to

$$
c_{i} \geq V_{m+1} / P \quad \text { for } i \epsilon N_{m}^{\mathbf{o}} .
$$

Conversely, if (6.28) holds, then any $\left(\mathbf{c}^{\mathbf{0}}, \mathbf{p}^{0}\right)$ satisfying (6.15-6.16), (6.27) and (6.29-6.31) obeys (6.36.5) and so is an equilibrium point. This completes the analysis for Case II.

We turn now to the situation

$$
V_{m}<P \quad \text { (Case III). }
$$

By (6.5) and (6.6),

$$
p_{i}^{0}=1 \quad \text { for } i \epsilon T \text {. }
$$

For $i \epsilon E$, if $p_{i}^{0}<1$ then (6.3) would imply $c_{i}^{0}=1$, which by (6.5) would imply $p_{i}^{0}=1$; hence

$$
p_{i}^{0}=1 \quad \text { for } i \epsilon E \text {. }
$$

According to (5.34), $U$ has been partitioned as $L \cup N_{m}^{0} \cup N_{m}^{-}$, where

$$
L=\left\{i: \quad V_{m}<V_{i}<P\right\}
$$

Also, $N_{m}^{+}$is partitioned as $T \cup E \cup L$. For $i \epsilon L$, if $c_{i}^{0}=1$ then (6.5) implies $p_{i}^{0}=1$, which by (6.4) implies $c_{i}^{\mathbf{0}}=0$. Hence

$$
c_{i}^{\mathbf{0}}<1 \quad \text { for } i \in L,
$$

which by (6.3) implies

$$
p_{i}^{\mathbf{o}} \geq V_{i} / P \quad \text { for } i \in L .
$$

For each of $\mathbf{c}^{\text {o }}$, let

$$
\begin{gathered}
M=\max \left\{V_{i} c_{i}^{\mathbf{0}}: \quad i \in U\right\}, \\
S=\left\{i: \quad i \in U, V_{i} c_{i}^{\mathbf{o}}=M\right\} .
\end{gathered}
$$


Then (6.34) and (6.5) imply

$$
c_{i}^{0} \geq M / P \quad \text { for } i \in E .
$$

If $|S| \leq m-|T \cup E|$, then (6.5) would imply $p_{i}^{0}=1$ for $i \epsilon S$, in which case (6.4) implies $c_{i}^{0}=0$ for $i \in S$, so that $M=0$ and $S=U$, which (since $m<n$ ) would contradict the supposition $|S| \leq m-$ $|T \cup E|$. Hence

$$
|T \cup E|+|S|>m \text {. }
$$

By (6.5) followed by (6.3),

$$
\begin{array}{ll}
p_{i}^{0}=0 & \text { for } i \in U-S, \\
c_{i}^{0}=1 & \text { for } i \in U-S .
\end{array}
$$

It follows from (6.43) and the definition of $M$ that

$$
M>\max \left\{V_{i}: \quad i \in U-S\right\}
$$

in particular, for each $s \in S, V_{s}$ exceeds that maximum. An immediate consequence is that

$$
L \text { is contained in } S \text {. }
$$

By (6.41), $S$ cannot consist just of $L$, and then (6.44) implies

$$
N_{m}^{\mathbf{o}} \text { is contained in } S \text {. }
$$

In particular $m \in S$, so that

$$
M=V_{m} c_{m}^{0}
$$

At this point it is convenient to consider separately the situation

$$
M=0, \quad \text { (Case IIIA) }
$$

or equivalently

$$
c_{i}^{0}=0 \quad \text { for } i \epsilon U \text {, }
$$

which by (6.3) requires

$$
p_{i}^{0} \geq V_{i} / P \quad \text { for } i \in U \text {, }
$$

implying

$$
m \geq|T \cup E|+\sum\left\{V_{i} / P: \quad i \epsilon U\right\} .
$$

Conversely, under Case III, if (6.51) holds, then there exist pairs $\left(\mathbf{c}^{\mathbf{0}}, \mathbf{p}^{\mathbf{0}}\right)$ satisfying (6.6), (6.49), (6.33$6.34)$ and (6.50); since any such pair obeys $(6.3-6.5)$, it is an equilibrium point. 
From now on, assume

$$
M>0 \quad \text { (Case IIIB). }
$$

Then it follows from (6.45) that $c_{i}^{0}>0$ for $i \epsilon L$, so that (6.4) implies $p_{i}^{0} \leq \mathrm{V}_{i} / \mathrm{P}$, which together with (6.37) yields

$$
p_{i}^{\mathbf{0}}=V_{i} / P \quad \text { for } i \epsilon L
$$

A similar argument using (6.46) yields

$$
p_{i}^{0} \leq V_{m} / P \quad \text { for } i \epsilon N_{m}^{o} .
$$

Also, (6.45) implies

$$
c_{i}^{\mathbf{0}}=V_{m} c_{m}^{\mathbf{0}} / V_{i} \quad \text { for } i \epsilon L,
$$

while (6.46) gives

$$
c_{i}^{\mathbf{0}}=c_{m}^{\mathbf{0}} \quad \text { for } i \epsilon N_{m}^{\mathbf{o}} .
$$

Combining (6.3) with (6.54) yields

$$
p_{i}^{\mathbf{o}}=V_{m} / P \quad \text { for } i \epsilon N_{m}^{0} \text {, if } c_{m}^{\mathbf{o}}<1
$$

Now partition $N_{m}^{-}$into $\beta$ blocks $B_{b}$, as described following (5.34). Then by (6.44), $S \cap N_{m}^{-}$ consists of the first $\alpha$ of these blocks, for some integer $\alpha$ with $0 \geq \alpha \geq \beta$. For each block $B_{b}$ in $S$, $\left\{c_{i}^{0}: \quad i \in B_{b}\right\}$ consists of a single number $c_{b}^{*}$, and

$$
c_{b}^{*}=c_{m}^{0} V_{m} / V_{b}^{*} \quad \text { for } 1 \leq b \leq \alpha .
$$

Since $c_{b}^{*} \leq 1,(6.58)$ yields

$$
V_{\alpha}^{*} \geq c_{m}^{0} V_{m}
$$

On the other hand, if $\alpha<\beta$, then (6.44) and (6.47) imply

$$
c_{m}^{\mathbf{0}}>V_{\alpha+1}^{*} / P \quad(\text { if } \alpha<\beta) .
$$

If $c_{m}^{0}=1$, this implies $\alpha=0$, and requires

$$
|T \cup E|+\sum\left\{V_{i} / P: \quad i \epsilon L\right\}+\left|N_{m}^{\mathbf{o}}\right| V_{m} / P \geq m .
$$

If $c_{m}^{0}<1$, then (6.58) implies $0<c_{b}^{0}<1$ for $1 \leq b<\alpha$, and thus by (6.3) and (6.4)

$$
p_{i}^{0}=V_{b}^{*} / P \quad \text { for } i \epsilon B_{b} \text { and } 1 \leq b<\alpha .
$$

Since $c^{*} \leq 1,(6.4)$ yields

$$
p_{i}^{0} \geq V_{\alpha}^{*} / P \quad \text { for } i \in B_{\alpha} \text {. }
$$


It follows from these results that

$$
\sum_{b=0}^{\alpha}\left|B_{b}\right| V_{b} / P \geq m-|T \cup E|-\sum\left\{V_{i} / P: \quad i \epsilon L\right\} \geq \sum_{b=0}^{\alpha-1}\left|B_{b}\right| V_{b}^{*} / P .
$$

Equality in the second relation is equivalent to equality in (6.63); this situation corresponds in the notation of (5.37) to $\lambda=\left|B_{\alpha}\right| V_{\alpha}^{*} / P$. If on the other hand $\lambda<\left|B_{\alpha}\right| V_{\alpha}^{*} / P$, then since

$$
\sum\left\{p_{i}^{0}: \quad i \in B_{\alpha}\right\}=\lambda
$$

by virtue of (6.33-6.34), (6.42), (6.52), (6.57) and (6.60), it follows that $p_{i}^{0}<V_{\alpha}^{*} / P$ must hold for some $i \in B_{\alpha}$, and so by (6.3) we have $c_{\alpha}^{*}=1$, which by (6.48) fixes the value of $c_{m}^{0}$ at $V_{\alpha}^{*} / V_{m}^{*}$.

Finally, observe that (6.51) and (6.64) will hold simultaneously if and only if the former holds as an equality while the latter's second part holds as an equality with $\alpha=\beta$.

\section{Formulation and Results for Model 3}

Recall that section 5 originally set out to analyze the consequences of the payoff functions

$$
\begin{aligned}
& F(\mathbf{c}, \mathbf{p})=\sum_{1}^{n}\left[V_{i}-P p_{i}\right] c_{i}, \\
& G(\mathbf{c}, \mathbf{p})=-\sum_{1}^{n} V_{i} c_{i}
\end{aligned}
$$

for inspectee and inspector respectively, but that this was found unfruitful within the framework of "equilibrium point" solutions to nonzero-sum games. The response in section 5 was to replace (7.2) with an alternative function (5.5) representing the loss (e.g., to consumers) due to undetected cheating (rather than all cheating). In this section we explore a different approach, in which (7.2) is retained but the customary game-theoretic framework is altered.

Specifically, the critical assumption here is that no matter what strategy $\mathbf{p}$ is selected by the inspector, the inspectee learns of it in advance (or can estimate it through experience), and so is able to select a strategy $\mathbf{c ( p )}$ which maximizes his payoff, i.e.,

$$
\mathrm{F}[\mathbf{c}(\mathbf{p}), \mathbf{p}]=\max _{\mathbf{c}} F(\mathbf{c}, \mathbf{p}) .
$$

(In general $\mathbf{p}$ is a mixed strategy, so that the inspectee need not know exactly which devices will be inspected but only the associated probabilities.) The inspector's problem, therefore, is to choose $\mathbf{p}$ so as to maximize the function

$$
H(\mathbf{p})=G[\mathbf{c}(\mathbf{p}), \mathbf{p}]
$$

To discuss this approach further, it is convenient to make the further hypothesis that each component of $\mathbf{c}(\mathbf{p})$ depends only on the corresponding component of $\mathbf{p}$, so that we can write $c_{i}\left(p_{i}\right)$ rather than $c_{i}(\mathbf{p})$. This hypothesis is reasonable per se (since the inspectee's $c_{i}$ 's are not linked by any constraints), and also is consistent with the consequences

$$
\begin{array}{ll}
c_{i}\left(p_{i}\right)=0 & \text { if } p_{i}>V_{i} / P, \\
c_{i}\left(p_{i}\right)=1 & \text { if } p_{i}<V_{i} / P
\end{array}
$$

of (7.1) and (7.3). 
In terms of the set ${ }^{2}$

$$
E(\mathbf{p})=\left\{i: \quad p_{i}=V_{i} / P\right\}
$$

we can identify a set of components of $\mathbf{c}(\mathbf{p})$, namely

$$
\left\{c_{i}\left(p_{i}\right): \quad i \in E(\mathbf{p})\right\}
$$

which are not determined by the criterion (7.3). That is, $\mathbf{c}(\mathbf{p})$ is not single-valued, and so the same is true of the inspector's objective function

$$
H(\mathbf{p})=-\sum_{1}^{n} V_{i} c_{i}\left(p_{i}\right)
$$

Some further assumption is needed to resolve this ambiguity. For example, since our critical assumption (that the inspectee knows $\mathbf{p}$ ) is "pessimistic" from the inspector's viewpoint, one might wish to be consistently pessimistic and thus to replace the right-hand side of (7.4) by its minimum over all $c(p)$ consistent with (7.3). This is equivalent to setting

$$
c_{i}\left(p_{i}\right)=1 \quad \text { all } i \epsilon E(\mathbf{p}) .
$$

At the opposite extreme, one might want to introduce a countervailing bias in the optimistic direction, and so would set

$$
c_{i}\left(p_{i}\right)=0 \quad \text { all } i \in E(\mathbf{p}) .
$$

This is the choice that will for the present be made. It involves a notion of "no cheating without a positive expectation of gain," which is not implausible but certainly involves appeal to considerations that are external to the model (and that, in particular, go beyond the penalty $P$ and its deterrent effect). After the consequences of hypothesis (7.9) have been analyzed, we will consider the more general situation in which this assumption is not imposed.

Retaining the notation

$$
V_{\mathrm{tot}}=\sum{ }_{1}^{n} V_{i},
$$

define

$$
V(I)=\sum\left\{V_{i}: \quad i \epsilon I\right\}
$$

for subsets $I$ of $N$, and set

$$
I(\mathbf{p})=\left\{i: \quad p_{i} \geq V_{i} / P\right\}
$$

Then $H(\mathbf{p})$ depends on $\mathbf{p}$ only via $I(\mathbf{p})$, since by (7.5), (7.6), (7.8) and (7.9),

$$
\begin{aligned}
H(\mathbf{p}) & =-\sum\left\{V_{i}: \quad i \epsilon N-I(\mathbf{p})\right\} \\
& =-V_{\mathrm{tot}}+V[I(p)] .
\end{aligned}
$$

\footnotetext{
${ }^{2}$ The following notation should not be confused with the usage of symbol " $E$ " in (3.10) or with the expected value function.
} 
Note that $I(\mathbf{p})$ corresponds to the set of devices on which cheating will not occur, according to (7.5) and (7.9). From (7.11) it follows that the inspector's problem can be written

choose $\mathbf{p}$ to maximize $V[I(\mathbf{p})]$.

In this context, it is natural to ask which subsets $I$ of $N$ can arise in the form $I(\mathbf{p})$ for some $\mathbf{p}$, i.e., as the "non-cheating" set for some strategy of the inspector. As will be shown in section 8, a set of conditions on a subset $I$ which are both necessary and sufficient that $I=I(\mathbf{p})$ for some $\mathbf{p}$, are

$$
\begin{gathered}
V(I) \leq P m, \\
I \subseteq \bar{T}=N-T, \\
P(m-|I|-|T|)<V(N-I-T) .
\end{gathered}
$$

For a subset $I$ of $N$ which obeys these three conditions, there may be many choices of $\mathbf{p}$ for which $I(\mathbf{p})=I$. If $|I|+|T|<m$, one such $\mathbf{p}$ is given by

$$
\begin{array}{ll}
p_{i}=1 & \text { for } i \epsilon I \cup T \\
p_{i}=\left[V_{i}(m-|I|-|T|)\right] / V(N-I-T) & \text { for } i \epsilon N-I-T .
\end{array}
$$

If $|I| \leq m \leq|I|+|T|$, then such a $\mathbf{p}$ is given by

$$
\begin{array}{ll}
p_{\mathrm{i}}=1 & \text { for } i \epsilon I, \\
p_{i}=[m-|I|] /|T| & \text { for } i \epsilon T, \\
p_{i}=0 & \text { for } i \epsilon N-I-T .
\end{array}
$$

If $|I|>m$ then such a $\mathbf{p}$ is given by

$$
\begin{array}{ll}
p_{i}=V_{i} / P+\left(1-V_{i} / P\right)[P m-V(I)] /[P|I|-V(I)] & \text { for } i \epsilon I, \\
p_{i}=0 & \text { for } i \epsilon N-I .
\end{array}
$$

(When $P|I|-V(I)=0$ then $V_{i}=P$ for all $i \epsilon I$ and we interpret (7.18) as $p_{i}=1$.) This will be proved in section 8 .

The inspector's problem can now be rephrased as that of choosing a subset $I$ of $N$, subject to (7.13) through (7.15), to

$$
\text { maximize } V(I) \text {. }
$$

When such an $I$ has been found, an optimal $\mathbf{p}$ can be calculated through (7.16), (7.17) or (7.18-7.19). For the maximization problem, (7.15) and (7.16) are, in fact, unnecessary, that is, if $I$ maximizes $V(I)$ subject to (7.13) and (7.14) then $|I|+|T| \geq m$, so that (7.16) does not arise, and $I$ satisfies (7.15). For a proof, suppose that $|I|+|T|<m$. Since $|\bar{T}|+|T|=n>m$, it follows that $I$ is a proper subset of $\bar{T}$. Let $j \in \bar{T}, j \notin I$, and set

$$
J=I \cup\{j\} .
$$


Since $|I| \leq|I|+|T|<m$, we have $|I| \leq m-1$ and so

$$
|J| \leq|J|+|T|=|I|+|T|+1 \leq m .
$$

Clearly $J$ satisfies (7.13) and (7.14) and the left-hand side of (7.15) is nonpositive. The left-hand side of (7.15) is equal to zero only when $|J|+|T|=m$ and, in this case, $J$ is a proper subset of $\bar{T}$, so that the right-hand side of (7.15) is positive. Hence $J$ satisfies (7.15) as well. This contradicts the assumption that $I$ maximizes $V(I)$ subject to (7.13-7.15).

For yet another formulation of the inspector's problem, introduce a vector $\mathbf{x}=\left(x_{1}, x_{2}, \ldots, x_{n}\right)$ of discrete variables

$$
x_{i}=\left\{\begin{array}{l}
1 \text { for } i \in I \\
0 \text { for } i \in N-I
\end{array}\right.
$$

Then the problem is to choose $\mathbf{x}$ to

$$
\operatorname{maximize} \sum_{I}^{n} V_{\mathrm{i}} x_{i}
$$

subject to the analogs of (7.13) and (7.14):

$$
\begin{gathered}
\sum_{1}^{n} V_{i} x_{i} \leq P m, \\
x_{i}=0 \quad \text { for } i \epsilon T .
\end{gathered}
$$

Problem (7.21)-(7.23) cannot in general be solved in closed form. As a (binary) integer program, it can (if $n$ is not too large) be solved by any of the methods developed for such problems. More specifically, it is a "knapsack problem," for which special algorithms are available. ${ }^{3}$ Its peculiarity as a knapsack problem, namely the presence of the same coefficients $\left(V_{i}\right)$ in both (7.21) and (7.22), suggest the possibility of more efficient "tailored" methods, but this will not be pursued here.

Example 1: Equal-Sized Firms. As before, we assume in this scenario that all $V_{i}=V$. If $P<$ $V$, then $T$ is all of $N$, so that (7.14) implies $I$ is empty. Thus (7.17) applies, giving

$$
p_{i}=m / n \quad \text { for all } i,
$$

and the associated payoffs are

$$
\begin{aligned}
& F^{\mathbf{0}}=V_{\mathrm{tot}}-P m, \\
& G^{\mathbf{0}}=-V_{\mathrm{tot}} .
\end{aligned}
$$

This case corresponds to weak social sanctions, resulting in cheating on every device.

If $P \geq V$, then $T$ is empty and the problem is that of choosing $I$ to maximize $|I|$ subject to

$$
|I| \leq P m / V
$$

To describe the solution in this case, let $k$ be the largest integer not exceeding either $P m / V$ or $n$, and let $I$ be any subset of $N$ with $|I|=k$. Since $n>m$ and $P \geq V$, it follows that $k \geq m$, so (7.18-7.19)

${ }^{3}$ See, e.g., A.V. Cabot, “An Enumeration Algorithm for Knapsack Problems," Operations Research 18 (1970), 306-311. 
applies to yield

$$
\begin{array}{ll}
p_{i}=m / k & \text { for } i \epsilon I, \\
p_{i}=0 & \text { for } i \epsilon N-I .
\end{array}
$$

The associated inspectee strategy is

$$
\begin{array}{ll}
c_{i}=0 & \text { for } i \in I, \\
c_{i}=1 & \text { for } i \in N-I,
\end{array}
$$

and the resultant payoffs are

$$
F^{\mathbf{0}}=-G^{0}=V(n-k)=V_{\mathrm{tot}}-V k
$$

See figure 5 for a depiction of the payoffs a functions of $P$ and $m . F^{0}$ is continuous along the line $P=V$, whereas $G^{\mathbf{0}}$ has a discontinuity across that line. To the right of this line, $F^{\mathbf{0}}$ and $G^{\mathbf{0}}$ are both discontinuous across each hyperbola $P m=t V$ for integers $t \leq n$, since the integer parameter $k$ changes value there.

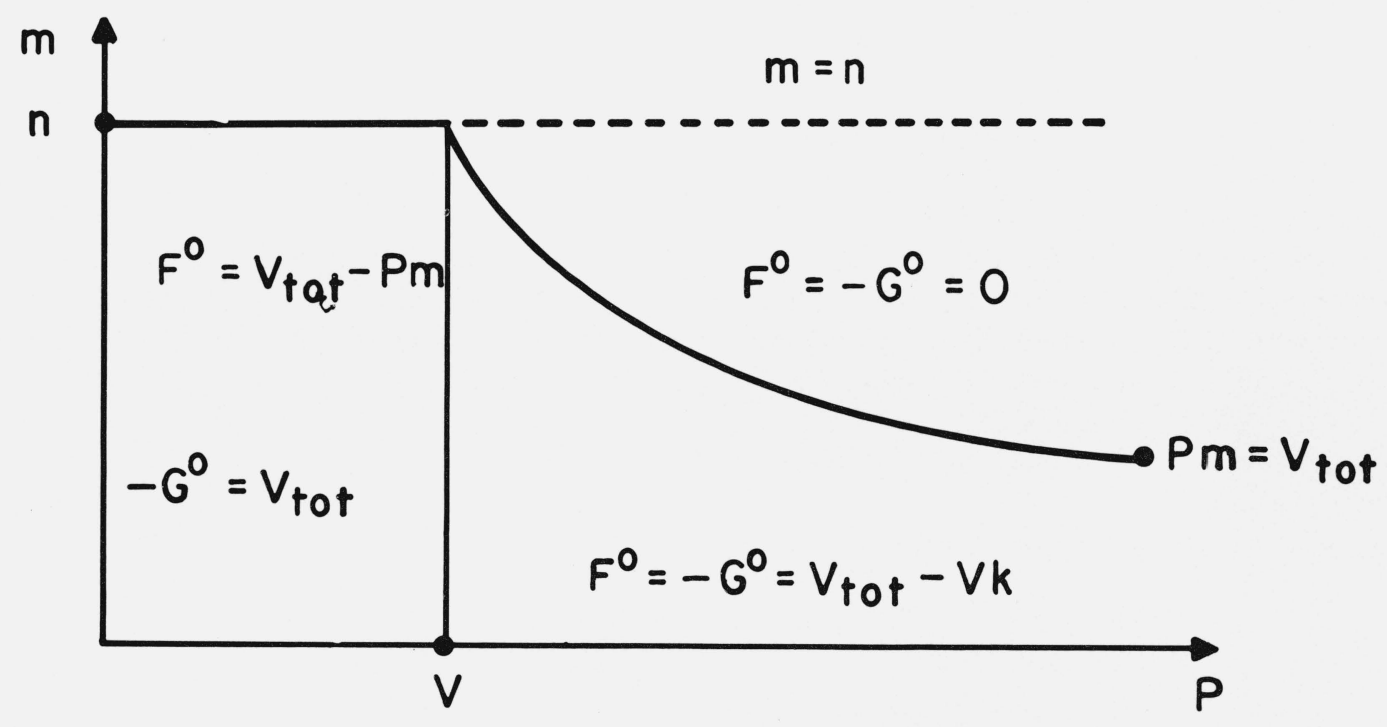

FIGURE 5. Net illicit gain $\left(F^{\circ}\right)$ and total illicit-activity level $\left(-G^{\circ}\right)$. (Equal-sized firms) ( $k$ is greatest integer $\leq P m / V)$.

Example 2: Big Firms, Little Firms. As before, $N$ is partitioned into nonempty subsets, $N=$ $B \cup S$, with

$$
\begin{array}{ll}
V_{i}=V_{b} & \text { for } i \epsilon B \\
V_{i}=V_{s} & \text { for } i \epsilon S,
\end{array}
$$

and $V_{b}>V_{s}$. The notations $n_{b}=|B|$ and $n_{s}=|S|$ will again be used. 
If $V_{b}>V_{s}>P$ then $T$ is all of $N$, so that (7.14) implies $I$ is empty (hence all $c_{i}=1$ ). Thus (7.17) applies, giving

$$
p_{i}=m / n \quad \text { for } i \epsilon N .
$$

The corresponding payoffs are

$$
F^{\mathrm{o}}=V_{\mathrm{tot}}-P m, \quad G^{\mathrm{o}}=-V_{\mathrm{tot}} .
$$

If $V_{b}>P \geq V_{s}$, then $T=B$ and, by (7.6), $c_{i}=1$ for all $i \epsilon B$. Thus by (7.14), $I$ must be a subset of $S$, and the inspector's problem is to choose such a subset to maximize $|I|$, subject to

$$
|I| \leq P m / V_{s}
$$

Two cases arise:

In case $n_{\mathrm{s}} \leq m$, we take $I=S$. This is clearly the largest possible subset of $S$, and it satisfies (7.29) since $n_{s} \leq \mathrm{m}$ and $V_{s} \leq P$. From (7.17)

$$
\begin{array}{ll}
p_{i}=1 & \text { for all } i \epsilon S, \\
p_{i}=\left(m-n_{s}\right) / n_{b} & \text { for all } i \epsilon B,
\end{array}
$$

and by (7.5) and (7.9), $c_{i}=0$ for all $i \epsilon S$. The resultant payoffs are

$$
F^{0}=-G^{0}=V_{b} n_{b}
$$

In the remaining case $m<n_{s}$, let $k$ be the largest integer not exceeding either $P m / V_{s}$ or $n_{s}$, and let $I$ be any subset of $S$ with $|I|=k$. (Thus $k=n_{s}$ and $I=S$ when $P m \geq V_{s} n_{s}$.) Since $P \geq V_{s}$ (and hence $P m / V_{s} \geq m$ ), and $n_{s}>m$, it follows that $k \geq m$, and so (7.18-7.19) applies to yield

$$
\begin{array}{ll}
p_{i}=m / k & \text { for } i \in I, \\
p_{i}=0 & \text { for } i \in N-I .
\end{array}
$$

Since $m / k \geq V / P$, it then follows from (7.5) and (7.9) that $c_{i}=0$ for $i \epsilon I$, while by (7.6), $c_{i}=1$ for $i \epsilon S-I$. The corresponding payoffs are

$$
F^{\mathrm{o}}=-G^{\mathrm{o}}=V_{\mathrm{tot}}-V_{s} k .
$$

Finally, if $P \geq V_{b}>V_{s}$, then $T$ is empty. Let $x_{b}=|I \cap B|$ and $x_{s}=|I \cap S|$. The inspector's problem is to choose integers $x_{b}$ and $x_{s}$, which

$$
\operatorname{maximize} z=V_{b} x_{b}+V_{s} x_{s}
$$

subject to

$$
\begin{gathered}
0 \leq x_{b} \leq n_{b}, \quad 0 \leq x_{s} \leq n_{s}, \\
V_{b} x_{b}+V_{s} x_{s} \leq P m
\end{gathered}
$$


If $P m \geq V_{\text {tot }}$ then the optimal $I$ is all of $N$ (i.e., $x_{b}=n_{b}$ and $x_{s}=n_{s}$ ), so that all $c_{i}=0$, implying $F^{\mathbf{o}}$ $=G^{\mathbf{o}}=0$, while (7.18) gives, for all $i$,

$$
p_{i}=V_{i} / P+\left(1-V_{i} / P\right)\left[\left(P m-V_{\mathrm{tot}}\right) /\left(P n-V_{\mathrm{tot}}\right)\right] .
$$

Now assume $P m<V_{\text {tot. }}$ No closed-form answer to (7.32-7.34) seems possible, but trial-anderror solution methods should suffice. The maximum value $z_{\max }$ in (7.32) can be estimated by

$$
P m-V_{b}<z_{\max } \leq P m
$$

here the right-hand inequality follows from (7.34), while the left-hand one follows by observing that if $z \leq P m-V_{b}$ then either $x_{b}$ can be increased by 1 (thus increasing $z$ by $V_{b}$ ) without violating (7.33) or (7.34), or else $x_{b}=n_{b}$ so that (since $\left.z \leq P m<V_{\mathrm{tot}}\right) x_{s}<n_{s}$, implying $x_{s}$ can be increased by 1 without violating the constraints. (If $P m \leq V_{s} n_{s}$, similar logic shows the left-hand side of (7.35) can be sharpened-i.e., increased-to $P m-V_{s}$.) Furthermore, any optimal solution must satisfy $x_{b}+x_{s}$ $\geq m$, since otherwise

$$
z_{\max }=V_{b} x_{b}+V_{s} x_{s} \leq V_{b}\left(x_{b}+x_{s}\right) \leq V_{b}(m-1) \leq P(m-1)=P m-P<P m-V_{b},
$$

contradicting (7.35). Thus $|I| \geq m$, so that (7.18-7.19) applies to yield

$$
p_{i}=V_{i} / P+\left(1-V_{i} / P\right)\left[P m-z_{\max }\right] /\left[P\left(x_{b}+x_{s}\right)-z_{\max }\right]
$$

for $I \epsilon I$, and $p_{i}=0$ otherwise. Thus $c_{i}=0$ for $i \epsilon I$ and $c_{i}=1$ otherwise, and the associated payoffs are

$$
F^{\mathbf{0}}=-G^{\mathbf{0}}=V_{b}\left(n_{b}-x_{b}\right)+V_{s}\left(n_{s}-x_{s}\right)=V_{\mathrm{tot}}-z_{\max },
$$

so that (7.35) yields the estimates

$$
V_{\mathrm{tot}}-P m \leq F^{0}=-G^{0}<V_{\mathrm{tot}}-P m+V_{b}
$$

(In accordance with a parenthetical remark above, the term $V_{b}$ in (7.38) can be reduced to $V_{s}$ if $P m$ $\leq V_{s} n_{s}$.)

Figure 6 shows the payoffs $F^{0}$ and $G^{0}$ as functions of $P$ and $m$. This concludes the discussion of Example 2 in the context of Model 3.

It remains to discuss the consequences of dropping assumption (7.9). For this purpose, we introduce the numbers

$$
c_{i}^{*}=c_{i}\left(V_{i} / P\right)
$$

which necessarily satisfy $0 \leq c_{i}^{*} \leq 1$, and also set

$$
N_{+}=\left\{i \in N: \quad c_{i}^{*}>0\right\} .
$$

In practice, it is unlikely that the $c_{i}^{*}$ 's (i.e., the probabilities of cheating at the various devices in a context of zero expected gain) would be known accurately. They might be assigned nominal high, medium or low values (such as $0.90,0.50$, and 0.10 ) in accordance with the insight or degree of optimism applicable to a specific application. They might well be assumed equal over all devices, or over each group of devices in a classification into a very few groups; these common values could then be varied in a parametric sensitivity analysis. At any rate, these numbers are treated as "given" in what follows. 


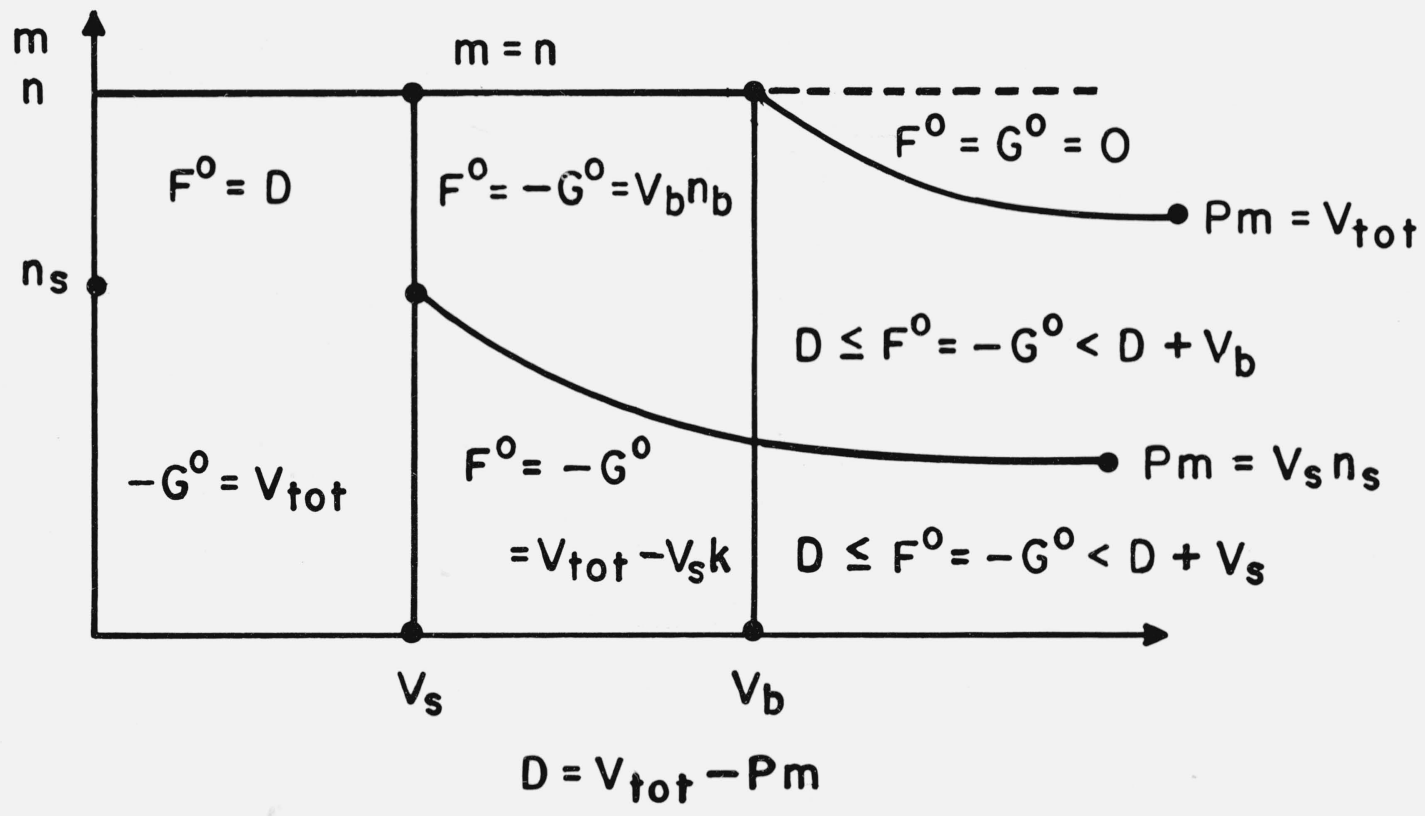

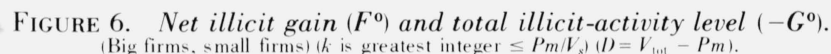

In addition to the set $E(\mathbf{p})$ defined in (7.7), it is convenient to define further sets

$$
\begin{gathered}
M(\mathbf{p})=\left\{i: \quad p_{i}>V_{i} / P\right\}, \\
L(\mathbf{p})=\left\{i: \quad P_{i}<V_{i} / P\right\},
\end{gathered}
$$

so that $M(\mathbf{p}), E(\mathbf{p})$ and $L(\mathbf{p})$ form a partition of $N$. By (7.5) and (7.6),

$$
\begin{array}{ll}
c_{i}=0 & \text { for } i \in M(\mathbf{p}), \\
c_{i}=1 & \text { for } i \in L(\mathbf{p}) .
\end{array}
$$

while by (7.39)

$$
c_{i}=c_{i}^{*} \quad \text { for } i \in E(\mathbf{p}) \text {. }
$$

It follows that

$$
-H(\mathbf{p})=\sum\left\{V_{i} c_{i}^{*}: \quad i \epsilon E(\mathbf{p}) \cap N_{+}\right\}+V[L(\mathbf{p})] .
$$

The members of the set $E(\mathbf{p}) \cap N_{+}$constitute the "violations" of the previous assumption (7.9). At this point, we temporarily restrict attention to the special case in which the set $E$ of (3.10) is empty, i.e., no $V_{i}$ is exactly equal to $P$. Then for $i \epsilon E(\mathbf{p})$, we have $p_{i}<1$. In this case, as will be shown in section 8 , such violations cannot occur for an optimal p unless the two conditions

$$
\begin{gathered}
M(\mathbf{p}) \text { is empty, } \\
p_{i}=0 \quad \text { for all } i \epsilon L(\mathbf{p})
\end{gathered}
$$


both hold. If they do hold, then

$$
\begin{aligned}
m & =\sum_{1}^{n} p_{i}=\sum\left\{p_{\mathrm{i}}: \quad i \in E(\mathbf{p})\right\} \\
& =\sum\left\{V_{i} / P: \quad i \in E(\mathbf{p})\right\}=V[E(\mathbf{p})] / P .
\end{aligned}
$$

Thus violations of (7.9) can only occur for an optimal $\mathbf{p}$ if the problem's data present the "coincidence" that some subset of the $V_{i}$ 's, specifically $\left\{V_{\mathrm{i}}: \quad i \in E(\mathbf{p})\right\}$, sums exactly to $P m$. In all other cases, the assumption (7.9) does not alter the problem of finding an optimal $\mathbf{p}$.

To describe how the exceptional cases just defined can be treated (and identified), define an inspector's strategy $\mathbf{p}$ to be exceptional if it satisfies (7.46) and (7.47), which as already seen, implies

$$
V[E(\mathbf{p})]=P m .
$$

Since $i \epsilon E(\mathbf{p})$ implies $V_{i} / P=p_{i} \leq 1$, it also follows that

$$
E(\mathbf{p}) \subseteq \bar{T}=N-T
$$

Conversely, if $E^{*}$ is any subset of $N$ such that

$$
V\left(E^{*}\right)=P m, \quad E^{*} \subseteq \bar{T},
$$

then $E^{*}=E(\mathbf{p})$ for one (in fact, precisely one) exceptional $\mathbf{p}$, namely the one defined by $p_{i}=V_{i} / P$ for $i \epsilon E^{*}$ and all other $p_{i}=0$. For exceptional $\mathbf{p}$ 's, (7.45) becomes

$$
\begin{aligned}
-H(\mathbf{p}) & =\sum\left\{V_{i} c_{i}{ }^{*}: \quad i \epsilon E(\mathbf{p})\right\}+V[n-E(\mathbf{p})] \\
& =\sum\left\{V_{i} c_{i}{ }^{*}: \quad i \in E(\mathbf{p})\right\}+V(N)-V[E(\mathbf{p})] \\
& =\sum\left\{V_{i} c_{i}{ }^{*}: \quad i \in E(\mathbf{p})\right\}+V_{\mathrm{tot}}-P m,
\end{aligned}
$$

so that the problem of selecting a "best" exceptional $\mathbf{p}$ is equivalent to that of choosing a subset $E^{*}$ of $N$ to

$$
\operatorname{minimize} \sum\left\{V_{i} c_{i}^{*}: \quad i \in E^{*}\right\}
$$

subject to (7.50). This can be written as a binary integer program in terms of variables

$$
y_{i}=\left\{\begin{array}{c}
1 \text { if } i \in E^{*} \\
0 \text { otherwise }
\end{array}\right.
$$

namely

$$
\operatorname{minimize} \sum{ }_{1}^{n} V_{i} c_{i}^{*} y_{\mathrm{i}}
$$

subject to

$$
\begin{aligned}
\sum{ }_{1}^{n} V_{i} y_{i} & =P m, \\
y_{i} & =0 \quad \text { if } i \epsilon T .
\end{aligned}
$$


As noted already, the preceding analysis leads (in particular) to those optimal p's which exhibit violations, i.e., for which $E(\mathbf{p}) \cap N_{+}$is nonempty. But it may be that no optimal $\mathbf{p}$ is of this type, and in any case the restriction to this type may unduly limit the variety of alternate optima found. Therefore, still assuming that $E$ is empty, we consider how to find optimal $\mathbf{p}$ which are violationfree.

For any such p, the first summand on the right side of (7.45) is zero, and since $L(\mathbf{p})$ and $I(\mathbf{p})$ are complementary subsets of $N$, the problem becomes that of choosing violation-free $\mathbf{p}$ to maximize $V[I(\mathbf{p})]$. This leads, as below (7.12), to the problem of choosing a subset $I$ of $N$ so as to maximize $V(I)$ subject to (7.13) through (7.15). As noted below (7.20), (7.15) is superfluous for this purpose and (7.16) does not arise.

Suppose that $|I| \leq m \leq|I|+|T|$. Then, by (7.17), no $i \epsilon I$ can lie in $E(\mathbf{p})$. Also, no $i \epsilon T$ can lie in $E(\mathbf{p})$ since $p_{i} \leq 1$ is required. If some $i \epsilon N-I-T$ lay in $E(\mathbf{p})$ then $V_{i}=0$, since $p_{i}=0$ by (7.17). Thus $\mathbf{p}$ is violation-free.

Next, suppose such an optimizing $I$ satisfies $|I|>m$. Since the emptiness of $E$ implies $1-V_{i} / P$ $\neq 0$, the $\mathbf{p}$ given by (7.18-7.19) has $E(\mathbf{p})$ empty and hence is violation-free, except if $V(I)=P m$ (i.e., if equality holds in (7.13)). So if $V(I)<P m$, then a comparison of $H(\mathbf{p})$ as given by (7.11) with the "exceptional-strategy maximum" of $H$ will identify the optimum.

Finally, suppose the maximum value of $V(I)$ is $P m$. (A higher value is forbidden by (7.13).) For any $I$ achieving this value, and any $\mathbf{p}$ for which $I(\mathbf{p})=I$, we have (since $V_{i} \leq P p_{\mathrm{i}}$ for all $i \in I(\mathbf{p})$ )

$$
\begin{gathered}
V[I(\mathbf{p})]=\sum\left\{V_{i}: \quad i \in I(\mathbf{p})\right\} \\
\leq P \sum\left\{p_{\mathrm{i}}: \quad i \in I(\mathbf{p})\right\} \leq P \sum_{1}^{n} p_{\mathrm{i}}=P m,
\end{gathered}
$$

and since the end terms in (7.55) are equal, equality must hold throughout, implying that $\mathbf{p}$ is given by

$$
\begin{array}{ll}
p_{i}=V_{i} / P & \text { for } i \in I, \\
p_{i}=0 & \text { for } i \in N-I .
\end{array}
$$

This $\mathbf{p}$ is exceptional, and will exhibit violations unless $I \cap N_{+}$is empty (for example, violations will occur if all $\left.c_{i}^{*}>0\right)$ ). If $I \cap N_{+}$is nonempty for all $I$ obeying (7.13-7.15) and yielding $V(I)=P m$, then finding a best violation-free $\mathbf{p}$ is not constrained by sharpening (7.13) to

$$
V(I)<P m .
$$

In this case, for any $I$ maximizing $V(I)$, it follows as before that the given by (7.16), (7.17) or (7.187.19) is violation-free and hence is a best violation-free strategy for the inspector.

In the context of the last paragraph, it is necessary to check whether the constraint (7.15) can still be omitted in maximizing $V(I)$. If $P m<V(\bar{T})$ then the justifying argument given below (7.20) remains valid. The same is true when $P m \geq V(\bar{T})$, except if $V(\bar{T})=V(U)=P m$. In this case (7.13) will be replaced by $\left(7.13^{\prime}\right)$ and the solutions will be all sets $I=\bar{T}-\{j\}$, where $j$ is such that $V_{j}=$ $\min \left\{V_{i}: \quad i \in \bar{T}\right\}$. Since $P m=V(\bar{T}),(7.15)$ is equivalent to $V(I)<P|I|+P|T|$, which is true when $|I|$ $\neq 0$ (since $I \subseteq \bar{T}$ ) and is also true when $|I|=0$ (since $|T|>0$ ).

ExAmPLE 1': Equal-Sized Firms. Here we illustrate the preceding material. The scenario (all $V_{i}$ $=V$ ) treated in Example 1 of this section is retained. However, the assumption (7.9) is no longer retained. It is assumed that $E$ is empty, i.e., $V \neq P$. "Violations" of (7.9) can occur only if $P m=k V$ for some integer $k$ with $k \leq n$. When no such $k$ exists, the analysis of Example 1 remains valid.

For the present example, we therefore assume that $P m=k V$ where $k$ is an integer with $k \leq n$. It is also necessary to specify the numbers $c_{i}^{*}$ of (7.39). Without loss of generality, the devices may 
be supposed numbered so that

$$
0 \leq c_{1}^{*} \leq c_{2}^{*} \leq \ldots \leq c_{n}^{*} \leq 1
$$

If $P<V$, then $T$ is all of $N$, so (7.54) implies that $E^{*}$ is empty. Thus no exceptions to (7.9) arise, so that the analysis in Example 1 is applicable.

Now suppose $P>V$. First a best exceptional strategy, $\mathbf{p}^{E}$, will be determined. Since $T$ is empty, (7.54) is vacuous. Since $P m / V=k$, problem (7.52-7.53) becomes that of choosing a subset $E^{*}$ of $N$ to minimize $\sum\left\{c_{i}^{*}: \quad i \in E^{*}\right\}$ subject to $\left|E^{*}\right|=k$. Clearly a solution is given by $E^{*}=\{1,2$, $\ldots, k\}$, and by the remark following (7.50), $\mathbf{p}^{E}$ is given by

$$
p_{i}{ }^{E}=V / P \quad \text { for } i \in E^{*}, \quad p_{i}^{E}=0 \text { otherwise. }
$$

Use of the formula preceding (7.51) gives

$$
H\left(\mathbf{p}^{E}\right)=-V_{\mathrm{tot}}+P m-V \sum_{1}^{k} c_{i}^{*} .
$$

Now a best violation-free strategy, $\mathbf{p}^{F}$, will be determined. The problem of maximizing $V(I)$, subject to (7.13-7.14), has as solutions all subsets $I$ of $N$ with $|I|=k$, implying $V(I)=k V=P m$. There are two subcases. If $I$ can be chosen disjoint from $N_{+}$, which is true if and only if $c_{k}^{*}=0$ in (7.58), then in particular $I$ can be taken as the set $E^{*}$ given above; here $\mathbf{p}^{E}$ is violation-free, can be taken as $\mathbf{p}^{F}$, and so is optimal. But if $\mathrm{c}_{k}^{*}>0$, then (7.13) must be replaced by $\left(7.13^{\prime}\right)$ in the maximization of $V(I)$. This problem has as solution any subset $I$ of $N$ with $|I|=k-1$ (note that $P>V$ and $P m=k V$ imply $k>m \geq 1$ ), in particular $I=\{1,2, \ldots, k-1\}$, yielding

$$
p_{i}^{F}=m /(k-1) \quad \text { for } i \epsilon I, \quad p_{i}^{F}=0 \text { otherwise. }
$$

Equation (7.11) yields

$$
H\left(\mathbf{p}^{F}\right)=-V_{\mathrm{tot}}+V(k-1)=-V_{\mathrm{tot}}+P m-V .
$$

When $c_{k}^{*}>0,(7.59)$ and (7.60) must be compared to determine the optimum. If $\sum_{1}^{k} c_{i}^{*} \geq 1$, then $H\left(\mathbf{p}^{E}\right) \leq H\left(\mathbf{p}^{F}\right)$ and so $\mathbf{p}^{F}$ is optimal. If $\sum_{1}^{k} c_{i}^{*} \leq 1$, then $H\left(\mathbf{p}^{E}\right) \geq H\left(\mathbf{p}^{F}\right)$ and $\mathbf{p}^{E}$ is optimal. This concludes the discussion of Example 1'.

To complete this discussion of Model 3, we must consider the consequences of removing the restriction, introduced shortly below (7.45), that $E$ is empty. Denote by $P^{*}$ the problem of choosing an inspector's strategy $\mathbf{p}$ to minimize the expression (7.45). For each integer $k$ in the range $0 \leq k \leq$ $\min \{|E|, m\}$, let $P_{k}$ be the problem obtained from $P^{*}$ by adjoining the constraints

$$
p_{i}=1 \quad \text { for exactly } k \text { members } i \epsilon E \text {. }
$$

If $\mathbf{p}^{k}$ denotes an optimal solution to $P_{k}$, and $K$ is such that

$$
H\left(\mathbf{p}^{K}\right)=\max \left\{H\left(\mathbf{p}^{K}\right): \quad 0 \leq k \leq \min (|E|, m)\right\},
$$

then clearly $\mathbf{p}^{K}$ is an optimal solution to $P^{*}$. Thus it suffices to be able to solve the problems $P_{k}$.

For each $k$, let $E_{k}$ be a subset of $E$ such that $|E|=k$ and $\sum\left\{c_{i}^{*}: I \epsilon E_{k}\right\}$ is minimum. In other words, this sum consists of the $k$ smallest members of $\left\{c_{i}^{*}\right.$ : i $\left.\epsilon \mathrm{E}\right\}$; ties can be broken arbitrarily. It will be shown in section 8 that $P_{k}$ has an optimal solution for which 


$$
\begin{array}{ll}
p_{i}=1 & \text { for } i \in E_{k}, \\
p_{\mathrm{i}}=\max \{0, m-k-(n-|E|)\} /(|E|-k) & \text { for } i \in E-E_{k} .
\end{array}
$$

Thus the analysis of $P_{k}$ can be confined to such strategies. Because $m<n,(7.64)$ gives $i \epsilon L(\mathbf{p})$, and so (7.45) yields

$$
\begin{aligned}
-H(\mathbf{p}) & =\sum\left\{V_{i} c_{i}^{*}: \quad i \epsilon E(p) \cap N_{+}-E\right\}+V[L(\mathbf{p})-E] \\
& +P\left[\sum\left\{c_{i}^{*}: \quad i \epsilon E_{k}\right\}+\left|E-E_{k}\right|\right] .
\end{aligned}
$$

Therefore the problem $P_{k}$ becomes that of choosing the nonnegative quantities $\left\{p_{i}: i \in N-E\right\}$, which by (7.63) and (7.64) must sum to $\min \{m-k, n-|E|\}$, so as to minimize the sum of the first two summands in (7.65). This problem, however, is of the type treated earlier (no $V_{\mathrm{i}}=P$ ), with $N$ replaced by $N-E$ and $m$ by $\min \{m-k, n-|\mathrm{E}|\}$, and in that sense ${ }^{4}$ can be regarded as "already solved."

It will also be shown in section 8 that the range of $k$, for problems $P_{k}$ to be treated as indicated above, can be contracted. To define the reduced range, let $U=\left\{i: V_{i}<P\right\}$ as before, and set

$$
\begin{aligned}
& \rho=\min \{|E|, m-l-V(U) / P\}, \\
& \sigma=\max \{-1, m-V(U) / P\} .
\end{aligned}
$$

Let $r$ be the greatest integer not exceeding $\rho$, and $s$ the smallest integer greater than $\sigma$. If $s \geq \min$ $\{|E|, m\}$, set $l$ equal to this minimum; otherwise, set $l$ equal to the greatest integer between $s$ and $\min \{|E|, m\}$ inclusive for which $\sum\left\{c_{i}^{*}: \quad i \in E_{l}-E_{s}\right\}<1$, with tie-breakings in the choices of $E_{l}-$ $E_{s}$ performed so that the latter is a subset of the former. Then the problems $P_{k}$ need be solved only over the range

$$
\max \{0, r\} \leq k \leq l \text {. }
$$

No loss of alternative optima is incurred by imposing the upper limit; the same is true of the lower limit, when $\left\{c_{i}^{*}: \quad i \in E\right\}$ has as many as $r$ members $<1$.

Example 1": Equal-Sized Firms. We can now complete the discussion of this scenario, for Model 3 without assumption (7.9), by covering the case excluded in the previous Example 1': that of all $V_{i}=P$. As before, we assume the numbering is such that

$$
0 \leq c_{1}^{*} \leq c_{2}^{*} \leq \ldots \leq c_{n}^{*} \leq 1
$$

and can therefore take $E_{k}=\{1,2, \ldots, k\}$. Since $E=N$, the first two terms on the right-hand side of (7.65) are zero, yielding

$$
H(\mathbf{p})=-P\left[\sum_{1}^{k} c_{i}^{*}+(n-k)\right]
$$

as the optimal value (call it $H_{k}$ ) for problem $P_{k}$. Because

$$
H_{k+1}-H_{k}=P\left(1-c_{k+1}^{*}\right) \geq 0,
$$

the value $K$ in (7.62) can be taken here to be $K=m$. Thus an optimal strategy for the inspector is

${ }^{4}$ Except for the possibility of equality in $\min (m-k, n-|E|)=m^{\prime} \leq n^{\prime}=n-|E|$, when the solution of the reduced problem is trivial. 
given, by (7.63) and (7.64), as

$$
\begin{array}{ll}
p_{i}=1 & \text { for } 1 \leq i \leq m, \\
p_{i}=0 & \text { for } m<i \leq n,
\end{array}
$$

and the optimum inspector's payoff is

$$
H_{m}=-P\left[\sum_{1}^{m} c_{i}^{*}+(n-m)\right]=-V_{\mathrm{tot}}+P m-V \sum_{1}^{m} c_{i}^{*}
$$

consistent with the result (7.59) for Example 1'.

In order to illustrate the various types of optimal strategies which can occur, we will close this section with a numerical example.

ExAmple 3: Let $n=5, m=2, P=1$, and

$$
V_{1}=V_{2}=1, V_{3}=0.3, V_{4}=V_{5}=0.5
$$

Thus $T$ is empty, $E=\{1,2\}$, and $V(U)=1$. Also, $\rho=-0.3$ and $r=-1$, so that the lower limit in (7.66) does not ease the analysis. Without loss of generality, we can assume $c_{1}^{*} \leq c_{2}^{*}$ and $c_{4}^{*} \leq c_{5}^{*}$. We have $\sigma=0.7$ and $s=1$, so that if $c_{2}^{*}=1$ then $l=1$ and so (7.66) would excuse us from analyzing problem $P_{2}$. The problems $P_{k}(k=0,1,2)$ will be considered separately.

For $P_{2}$ we have $E_{2}=\{1,2\}$. By (7.63),

$$
p_{1}=p_{2}=1,
$$

and, in the reduction of $P_{2}$ to devices $\left\{D_{3}, D_{4}, D_{5}\right\}$, we have $n^{\prime}=3$ and $m^{\prime}=0$. Clearly this has the sole solution

$$
p_{3}=p_{4}=p_{5}=0
$$

Combining these, we get the unique optimal solution to $P_{2}$. By (7.65),

$$
-H_{2}=1.3+c_{1}^{*}+c_{2}^{*} \text {. }
$$

For $P_{1}$ we can take $E_{1}=\{1\}$. By (7.63-7.64),

$$
p_{1}=1, \quad p_{2}=0,
$$

and, in the reduction of $P_{1}$ to $\left\{D_{3}, D_{4}, D_{5}\right\}$ we have $n^{\prime}=3$ and $m^{\prime}=1$. According to (7.51) through (7.54), finding a best exceptional strategy $\mathbf{p}^{E}$ for this reduced problem is equivalent to finding $\left\{y_{3}\right.$, $\left.y_{4}, y_{5}\right\}$, each either 0 or 1 , to

$$
\text { minimize } 0.3 c_{3}^{*} y_{3}+0.5 c_{4}^{*} y_{4}+0.5 c_{5}^{*} y_{5}
$$

subject to

$$
0.3 y_{3}+0.5 y_{4}+0.5 y_{5}=1 \text {. }
$$

The only solution of the constraint in $(0,1)$-valued variables is $y_{3}=0, y_{4}=y_{5}=1$, yielding

$$
p_{3}^{E}=0, \quad p_{4}^{E}=p_{5}^{E}=1 / 2,
$$


and corresponding via (7.65) to an $H$-value given by

$$
-H_{1}^{E}=1.3+c_{1}^{*}+0.5 c_{4}^{*}+0.5 c_{5}^{*} .
$$

According to (7.21) through (7.23) finding a best violation-free strategy $p^{F}$ for the reduced problem begins by finding $\left\{x_{3}, x_{4}, x_{5}\right\}$, all 0 or 1 , to

$$
\operatorname{maximize} \quad 0.3 x_{3}+0.5 x_{4}+0.5 x_{5}
$$

subject to

$$
0.3 x_{3}+0.5 x_{4}+0.5 x_{5} \leq 1 .
$$

Since the maximum achieves equality in (7.69), if $c_{5}^{*}>0$ then (7.13) is to be replaced by (7.13'), i.e., (7.69) is to be replaced by

$$
0.3 x_{3}+0.5 x_{4}+0.5 x_{5}<1 \text {. }
$$

This yields alternate solutions $I=\{3,4\}$ and $I=\{3,5\}$; the first of them gives, by (7.18-7.19),

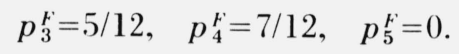

By (7.65),

$$
-H_{1}^{F}=1.5+c_{1}^{*} .
$$

Comparison of (7.68) and (7.69) yields

$$
\begin{array}{ll}
H_{1}^{E}=\max \left\{H_{1}^{E}, H_{1}^{F}\right\} & \text { if } c_{4}^{*}+c_{5}^{*} \leq 0.41 \\
H_{1}^{F}=\max \left\{H_{1}^{E}, H_{1}^{F}\right\} & \text { if } c_{4}^{*}+c_{5}^{*} \geq 0.41 .
\end{array}
$$

Finally, corresponding to problem $P_{0}$ we have the empty set $E_{0}$. By (7.64),

$$
p_{1}=p_{2}=0 \text {. }
$$

The reduction of $P_{0}$ to $\left\{D_{3}, D_{4}, D_{5}\right\}$ has $n^{\prime}=3$ and $m^{\prime}=2$. Since no subset of $\left\{V_{3}, V_{4}, V_{5}\right\}$ sums to $\mathrm{Pm}^{\prime}$, we need only find the best violation-free strategy for this reduced problem. This leads to $I=$ $\{3,4,5\}$, and by $(7.18-7.19)$,

$$
p_{3}=10 / 17, \quad p_{4}=p_{5}=12 / 17 \text {. }
$$

By (7.65),

$$
-H_{0}=2
$$

The maximum value $H_{\max }$ of $H$, corresponding to the optimal inspector's strategy, is given by

$$
\begin{aligned}
-H_{\max } & =\min \left\{-H_{2},-H_{1}^{E},-H_{1}^{F},-H_{0}\right\} \\
& =\min \left\{1.3+c_{1}^{*}+c_{2}^{*}, 1.3+c_{1}^{*}+0.5\left(c_{4}^{*}+c_{5}^{*}\right), 1.5+c_{1}^{*}, 2\right\} .
\end{aligned}
$$


Note that the value of $c_{3}^{*}$ is irrelevant. In table 3, it is shown that each of the four possible optimal strategies described above, and correspondingly each of the four candidate expressions for the minimum in (7.72), can yield the optimum for some choices of $\left\{c_{1}^{*}, c_{2}^{*}, c_{4}^{*}, c_{5}^{*}\right\}$. In each row of the table, the optimal value of $H$ is marked with an asterisk.

TABLE 3. Alternative forms of the optimum for Example 3

\begin{tabular}{|c|c|c|c|c|}
\hline & \multirow{2}{*}{$\begin{array}{l}\text { Problem } P_{2} \\
p_{1}=p_{2}=1\end{array}$} & \multicolumn{2}{|r|}{ Problem $P_{1}$} & \multirow{2}{*}{$\begin{array}{l}\text { Problem } P_{0} \\
p_{1}=p_{2}=0\end{array}$} \\
\hline & & $p_{1}=1$ & $p_{2}=0$ & \\
\hline & $p_{3}=p_{4}=p_{5}=0$ & $p_{3}^{E}=0 p_{4}^{E}=p_{5}^{E}=\frac{1}{2}$ & $p_{3}^{F}=5 / 12, p_{4}^{F}=7 / 12, p_{5}^{F}=0$ & $\begin{array}{l}p_{3}=10 / 17 \\
p_{4}=p_{5}=12 / 17\end{array}$ \\
\hline $\begin{array}{l}c_{1}^{*}=c_{2}^{*}=0.1 \\
c_{4}^{*}=c_{5}^{*}=0.9\end{array}$ & $-1.5^{*}$ & -2.3 & -1.6 & -2 \\
\hline $\begin{array}{l}c_{1}^{*}=0.1, c_{2}^{*}=0.9 \\
c_{4}^{*}=c_{5}^{*}=0.1\end{array}$ & -2.3 & $-1.5^{*}$ & -1.6 & -2 \\
\hline $\begin{array}{l}c_{1}^{*}=0.1, c_{2}^{*}=0.9 \\
c_{4}^{*}=c_{5}^{*}=0.9\end{array}$ & -2.3 & -2.3 & $-1.6^{*}$ & -2 \\
\hline $\begin{aligned} c_{1}^{*} & =c_{2}^{*}=c_{4}^{*} \\
& =c_{5}^{*}=0.9\end{aligned}$ & -3.1 & -3.1 & -2.4 & $-2^{*}$ \\
\hline
\end{tabular}

\section{Verifications for Model 3}

In this section we present proofs of several assertions made during the analysis of Model 3 in section 7 .

For the first of these, recall from (7.10) the definition

$$
I(\mathbf{p})=\left\{i: \quad p_{i} \geq V_{i} / P\right\} .
$$

We are to show that a subset $I$ of $N$ has the form $I(\mathbf{p})$, for at least one $\mathbf{p}$, if and only if $I$ satisfies the three conditions

$$
\begin{gathered}
V(I) \leq P m, \\
I \subseteq \bar{T}=N-T, \\
P(m-|I|-|T|)<V(N-I-T) .
\end{gathered}
$$

For the proof of sufficiency, suppose $I$ obeys (8.2-8.4). If $|I|+|T|<m$ then, following (7.16), set

$$
\begin{array}{ll}
p_{i}=1, & \text { for } i \epsilon I \cup T, \\
p_{i}=\left[V_{i}(m-|I|-|T|)\right] / V(N-I-T) & \text { for } i \epsilon N-I-T
\end{array}
$$

Since $m<n$ and all $V_{i}>0$, the denominator in (8.6) is strictly positive. It is clear that all $p_{i} \geq 0$, and it follows from (8.4) and (8.6) that $p_{i}<1$ for $i \epsilon N-I-T$, so that all $p_{i} \leq 1$. From (8.6) we have

$$
\sum\left\{p_{i}: \quad i \in N-I-T\right\}=m-|I|-|T|,
$$


so that $\sum_{1}^{n} p_{i}=m$. Thus (8.5-8.6) indeed define a proper p. It follows from (8.3) and (8.5) that $i \in I$ implies $\mathrm{i} \epsilon I(\mathbf{p})$. It follows from (8.6) and (8.4) that $i \epsilon N-I$ implies $i \epsilon N-I(\mathbf{p})$. So $I=I(\mathbf{p})$, as desired.

$$
\text { If }|I| \leq m \leq|I|+|T| \text {, then following (7.17), set }
$$

$$
\begin{array}{ll}
p_{i}=1 & \text { for } i \epsilon I, \\
p_{i}=[m-|I|] /|T| & \text { for } i \epsilon T, \\
p_{i}=0 & \text { for } i \epsilon N-I-T .
\end{array}
$$

The denominator of (8.8) can be nonpositive only when $T$ is empty. Clearly $0 \leq p_{i} \leq 1$ for all $i$. Also, 넴 $p_{i}=m$. Thus, (8.7-8.9) define a proper p. Clearly (8.7) implies that $i \in I(\mathbf{p})$ for all $i \in I$ and, if $i \in N-I$, then (8.8-8.9) imply that $i \in N-I(\mathbf{p})$. So $I=I(p)$, as desired.

If $|I|>m$ then, following (7.18-7.19), set

$$
\begin{array}{ll}
p_{i}=V_{i} / P+\left(1-V_{i} / P\right)[P m-V(I)] /[P|I|-V(I)] & \text { for } i \epsilon I, \\
p_{i}=0 & \text { for } i \epsilon N-I .
\end{array}
$$

In view of (8.2), the denominator of (8.10) could be nonpositive only if $P|I| \leq P m$, contradicting $|I|$ $>m$. It follows, using (8.10), (8.2), and (8.3), that all $p_{i} \geq 0$. Using $|I|>m$, it follows from (8.10) that $p_{i} \leq 1$ for $i \in I$, and thus for all $i$. Also

$$
\begin{aligned}
\sum_{1}^{n} p_{i} & =V(I) / P+[|I|-V(I) / P][P m-V(I)] /[P|I|-V(I)] \\
& =V(I) / P+(1 / P)[P m-V(I)]=m .
\end{aligned}
$$

Thus (8.10-8.11) define a proper p. Clearly (8.11) implies that $i \epsilon N-I(\mathbf{p})$ for all $i \epsilon N \geq I$. And if $i \in I$, then (8.10) together with the previous reasoning, implies that $p_{i} \geq V_{i} / P$, assuring $i \in I(\mathbf{p})$. Thus again $I=I(\mathbf{p})$, as desired.

For the necessity proof, we show that each set $I(\mathbf{p})$ satisfies (8.2-8.4). First,

$$
\begin{aligned}
V[I(\mathbf{p})]=\sum\left\{V_{i}: \quad i \in I(\mathbf{p})\right\} & \leq \sum_{P}\left\{P p_{i}: \quad i \in I(p)\right\} \\
& \leq P \sum_{1}^{n} p_{i}=P m
\end{aligned}
$$

verifying (8.2). Second, by (8.1), $i \in I(\mathbf{p})$ implies $1 \geq V_{i} / P$, verifying (8.3). Third,

$$
\begin{aligned}
m=\sum_{1}^{n} p_{i} & =\sum\left\{p_{i}: \quad i \epsilon \bar{T}-I(\mathbf{p})\right\}+\sum\left\{p_{i}: \quad i \epsilon I(\mathbf{p})\right\}+\sum\left\{p_{i}: \quad i \epsilon T\right\} \\
& \leq \sum\left\{V_{i} / P: \quad i \epsilon \bar{T}-I(\mathbf{p})\right\}+|I(\mathbf{p})|+|T| \\
& =\{V(\bar{T})-V[I(\mathbf{p})]\} / P+|I(\mathbf{p})|+|T|,
\end{aligned}
$$

with strict inequality when $\bar{T}-I(\mathbf{p})$ is nonempty. This verifies (8.4) unless $I(\mathbf{p})=T$ in which case (8.4) follows from $n>m$.

Next suppose $\mathbf{p}$ is an optimal strategy for the inspector, that $E=\left\{i: V_{i}=P\right\}$ is empty, and that

$$
E(\mathbf{p}) \cap N_{+}=\left\{i: \quad p_{i}=V_{i} / P \text { and } c_{i}^{*}>0\right\}
$$

is nonempty. We are to prove that

$$
M(\mathbf{p})=\left\{j: \quad p_{j}>V_{j} / P\right\} \text { is empty. }
$$


and that

$$
p_{k}<V_{k} / P \text { implies } p_{k}=0 \text {. }
$$

For the proof, first observe that $p_{i}<1$ (i.e., $V_{i}<P$ ) for each $i \epsilon E(\mathbf{p}) \cap N_{+}$. If there existed $j \in M(\mathbf{p})$, then one could choose a number $\delta$ with

$$
0<\delta<\min \left\{1-V_{i} / P, p_{j}-V_{j} / P\right\}
$$

and then define an inspector's strategy $\mathbf{q}$ by

$$
q_{i}=p_{i}+\delta, \quad q_{j}=p_{j}-\delta, \quad q_{t}=p_{t} \text { for } t \neq i, j .
$$

Since $M(\mathbf{q})=M(\mathbf{p}) \cup\{i\}, E(\mathbf{q})=E(\mathbf{p})-\{i\}$ and $L(\mathbf{q})=L(\mathbf{p})$, it follows from (7.45) that

$$
-H(\mathbf{q})=-H(\mathbf{p})-V_{\mathrm{i}} c_{i}^{*}<-H(\mathbf{p}),
$$

contradicting the optimality of p. Hence (8.10) holds. If there existed an index $k$ for which (8.14) was contradicted, i.e., $k \in L(\mathbf{p})$ and $p_{k}>0$, then one could choose a number $\eta$ with

$$
0<\eta<\min \left\{1-V_{i} / P, p_{k}\right\}
$$

and then define an inspector's strategy $\mathbf{r}$ by

$$
r_{i}=p_{i}+\eta, \quad r_{k}=p_{k}-\eta, \quad r_{t}=p_{t} \text { for } t \neq i, k
$$

As above, this would lead to a contradiction of the optimality of $\mathbf{p}$, so (8.14) must hold.

It remains to verify the assertions made in the final part of section 7, that following the discussion of Example $1^{\prime}$. Recall that $P^{*}$ is the problem of choosing an inspector's strategy $\mathbf{p}$ to minimize

$$
\begin{aligned}
-H(\mathbf{p}) & =\sum\left\{V_{i} c_{i}^{*}: \quad i \in E(\mathbf{p})\right\}+V[L(\mathbf{p})] \\
& =\sum\left\{V_{i} c_{i}^{*}: \quad i \in E(\mathbf{p})-E\right\}+V[L(\mathbf{p})-E] \\
& +P\left[\sum\left\{c_{i}^{*}: \quad i \in E(\mathbf{p}) \cap E\right\}+|L(p) \cap E|\right] .
\end{aligned}
$$

For $0 \leq k \leq \min \{|E|, m\}, P_{k}$ is the problem of minimizing $H$ subject to the further restriction that $|E(\mathbf{p}) \cap E|=k$.

The first of these assertions is that $P_{k}$ has an optimal solution in which $E(\mathbf{p}) \cap E=E_{k}$, where $E_{k}$ is any particular solution to the problem of finding a subset $E^{\prime}$ of $E$ which minimizes $\Sigma$ $\left\{c_{i}^{*}: i \epsilon E^{\prime}\right\}$ subject to $\left|E^{\prime}\right|=k$. In fact, the following argument will show that every optimal solution $\mathbf{p}$ of $P_{k}$ is such that $E$ ( $\left.\mathbf{p}\right) \cap E$ is a solution of the last-mentioned problem, which we denote $Q_{k}$.

For the proof, assume $\mathbf{p}$ is an optimal solution of $P_{k}$ for which $E(\mathbf{p}) \cap E \neq E_{k}$. Since these two sets each have $k$ members, it follows that $0<k<|E|$ and that there exist indices

$$
i \epsilon E_{k}-E(\mathbf{p}) \cap E, \quad j \epsilon E(\mathbf{p}) \cap E-E_{k} \text {. }
$$

By the definition of $E_{k}$, we have $c_{i}^{*} \leq c_{j}^{*}$, with strict inequality if $E(\mathbf{p}) \cap E$ is not a solution of $Q_{k}$ (i.e., does not correspond to $k$ smallest members of the indexed set $\left\{c_{r}^{*}: r \in E\right\}$ ). Define an inspector's strategy $\mathbf{q}$ by setting

$$
q_{j}=p_{i}<1, \quad q_{i}=p_{j}=1, \quad q_{t}=p_{\mathrm{t}} \quad \text { for } t \neq i, j .
$$


Then $E(\mathbf{q})-E=E(\mathbf{p})-E, L(\mathbf{q})-E=L(\mathbf{p})-E$, and

$$
\begin{aligned}
& E(\mathbf{q}) \cap E=[E(\mathbf{p}) \cap E-\{j\}] \cup\{i\}, \\
& L(\mathbf{q}) \cap E=[L(\mathbf{p}) \cap E-\{i\}] \cup\{j\} .
\end{aligned}
$$

Thus q satisfies the condition defining $P_{k}$, and it follows from (8.15) that

$$
-H(\mathbf{p})-[-H(\mathbf{q})]=P\left(c_{j}-c_{i}\right) \geq 0,
$$

where the inequality is strict if $E$ (p) $\cap E$ is not a solution of $Q_{k}$, contradicting the optimality of $\mathbf{p}$ for $P_{k}$. Thus equality must hold, and so $\mathbf{q}$ is also optimal for $P_{k}$; repetition of this step will clearly lead to an optimal strategy $\pi$ for $P_{k}$ such that $E(\pi) \cap E=E_{k}$, as desired.

We are now assured that $P_{k}$ has an optimal strategy $\mathbf{p}$ such that

$$
\begin{array}{ll}
p_{i}=1 & \text { for } i \epsilon E_{k}, \\
p_{i}<1 & \text { for } i \epsilon E-E_{k} .
\end{array}
$$

By (8.15), for such a strategy $H(\mathbf{p})$ does not depend on the specific values of $\left\{p_{i}\right.$ : $\left.i \epsilon E-E_{k}\right\}=$ $\left\{p_{i}: \quad i \in L(\mathbf{p}) \cap E\right\}$. To see how these values might be chosen, note that by (8.16), the devices $\left\{D_{i}: i \in E_{k}\right\}$ receive an amount $k$ from the total $\sum_{1}^{n} p_{i}=m$ of "inspection resources." For an optimal solution to $P_{k}$, since $H(\mathbf{p})$ does not depend on $\left\{p_{i}\right.$ : $\left.i \in E-E_{k}\right\}$ so long as they are $<1$, it can certainly do no harm to make available to the remaining $n-|E|$ devices $\left\{D_{i}\right.$ : $\left.i \epsilon N-E\right\}$ as much of the remaining subtotal $m-k$ of inspection resources as they can absorb. This amount

$$
m-k-\min \{m-k, n-|E|\}=\max \{0, m-k-(n-|E|)\}
$$

is to be realized as $\sum\left\{p_{i}\right.$ : $\left.i \epsilon E-E_{k}\right\}$, subject to (8.17). The simplest such allocation of this residual is the uniform one, leading to (7.63) and (7.64); alternative allocations lead to alternative optimal solutions to $P_{k}$.

For the verification of (7.66), first observe that problem $P_{k}$ has been reduced to that of choosing the probabilities $\left\{p_{\mathrm{i}}\right.$ : $\left.i \in N-E=U \cup T\right\}$, summing to $\min \{m-k,|U \cup T|\}$, so as to minimize the function given by (7.65), namely

$$
\begin{aligned}
-H(\mathbf{p}) & =\sum\left\{V_{i} c_{i}^{*}: \quad i \epsilon E(\mathbf{p})-E\right\}+V[L(\mathbf{p})-E] \\
& +P\left[\sum\left\{c_{i}^{*}: \quad i \in E_{k}\right\}+\left|E-E_{k}\right|\right] .
\end{aligned}
$$

Let $H_{k}$ denote the corresponding maximum value of $H$. The solution of the original problem, $P^{*}$, has been reduced to determining an index $k$ for which $\max \left\{H_{k}\right.$ : $\left.0 \leq k \leq m\right\}$ occurs, and solving the associated problem $P_{k}$.

The set-inclusions

$$
E(\mathbf{p})-E \subseteq U, \quad T \subseteq L(\mathbf{p})-E
$$

are easily verified. Their use shows that (8.18) can be written

$$
\begin{gathered}
-H(\mathbf{p})=\sum\left\{V_{i} c_{i}^{*}: \quad i \epsilon E(\mathbf{p}) \cap U\right\}+V[L(\mathbf{p}) \cap U] \\
+V(T)+P\left[\sum\left\{c_{i}^{*}: \quad i \epsilon E_{k}\right\}+\left|E-E_{k}\right|\right] .
\end{gathered}
$$


Thus solving $P_{k}$ is equivalent to choosing the probabilities $\left\{p_{i}: i \epsilon U\right\}$, summing to $\min \{m-k$, $|U|\}$, so as to minimize the sum of the first two summands on the right-hand side in (8.19). The probabilities $\left\{p_{i}: \quad i \in T\right\}$ can be chosen arbitrarily, except that they must sum to $\min \{m-k$, $|U \cup T|\}-\min \{m-k,|U|\}$, which can be achieved since the difference lies between 0 and $|T|$ inclusive.

Let $r$ be the largest integer not greater than

$$
\rho=\min \{|E|, m-1-V(U) / P\},
$$

and assume for this and the next paragraph that $\rho>0$, so that $r \geq 0$. Let $\mu=\min (|E|, m)$. We will next show that

$$
\max \left\{H_{k}: r \leq k \leq \mu\right\}=\max \left\{H_{k}: 0 \leq k \leq \mu\right\} .
$$

In other words, the search for the maximum in (7.62) can be confined to the subrange $\{k: \quad r \leq k \leq$ $\mu\}$ of $\{k: 0 \leq k \leq \mu\}$. Note that this is precisely the assertion made by the first equality in (7.66). The argument will also show that if $r$ members of $\left\{c_{i}^{*}: \quad \mathrm{i} \epsilon \mathrm{E}\right\}$ are $<1$, then no optima exist outside this subrange, i.e.,

$$
\max \left\{H_{k}: 0 \leq k<r\right\}<\max \left\{H_{k}: r \leq k \leq \mu\right\} .
$$

For the proof, assume $k \leq r$. Since $r \leq \rho$, it follows that $k<m-V(U) / P$. Moreover, since $V_{i}$ $<P$ for $i \in U$, it follows that $V(U) / P<|U|$ if $U$ is nonempty. Thus, if $U$ is nonempty, then

$$
V(U) / P<\min \{m-k,|U|\} \leq|U| .
$$

This implies that the probabilities $\left\{p_{i}: \quad i \in U\right\}$, summing to $\min \{m-k,|U|\}$, can be chosen so that $p_{i}>V_{i} / P$. Such a choice (vacuously possible if $U$ is empty) makes the first two summands on the right-hand side of (8.19) vanish, and therefore achieves the optimum in problem $P_{k}$. It follows that

$$
H_{k}=-V(T)-P\left[S_{k}+\left|E-E_{k}\right|\right] \quad \text { for } k \leq r,
$$

where $S_{k}$ denotes the sum of $k$ smallest members of $\left\{c_{i}^{*}: \quad \mathrm{i} \in \mathrm{E}\right\}$. Thus, for $k<r$,

$$
H_{k+1}-H_{k}=P\left[1-\left(S_{k+1}-S_{k}\right)\right] \geq 0,
$$

where the inequality holds because $S_{k+1}-S_{k}$ is equal to some $c_{i}^{*}$. Moreover, if each summand of $S_{r}$ is $<1$, then for $k=r-1$ this inequality must be strict. The results (8.21) and (8.22) follow immediately from these observations.

Next let $s$ be the smallest integer greater than

$$
\sigma=\max \{-1, m-V(U) / P\}
$$

and consider any $k$ with $k \leq \mu$ and

$$
\sum\left\{c_{i}^{*}: \quad i \in E_{k}-E_{s}\right\} \geq 1
$$

It will be shown below that $H_{k}<H_{s}$. This implies the second inequality of (7.66), completing the verification of the assertions in section 7. In (8.25), it is assumed that tie-breaking in the formation of $E_{k}$ and $E_{s}$ is performed so that the latter is a subset of the former; that is, the sum in (8.25) consists 
of $k-s$ largest among $k$ smallest members of $\left\{c_{i}^{*}: i \epsilon E\right\}$, and is equal to $S_{k}-S_{s}$ in the notation of the preceding paragraph. If $s=0, E_{s}$ is the empty set.

The proof that $H_{k}<H_{s}$ will consist of showing that

$$
-H_{k} \geq V(U)-P(m-k)+V(T)+P\left[S_{k}+|E|-k\right],
$$

and that

$$
-H_{s}<V(U)-P(m-s-1)+V(T)+P\left[S_{s}+|E|-s\right] .
$$

These inequalities together imply

$$
\begin{aligned}
\left(-H_{s}\right)-\left(-H_{k}\right) & <P(s+1-k)+P\left[S_{s}-S_{k}-(s-k)\right] \\
& =P\left[1-\left(S_{k}-S_{s}\right)\right] \leq 0
\end{aligned}
$$

where the last inequality follows from (8.25); this yields $H_{s}>H_{k}$.

To prove (8.26), let $\mathbf{p}^{k}$ be an optimal strategy for $P_{k}$, so that (8.19) yields

$$
\begin{gathered}
-H_{k} \geq V\left[L\left(\mathbf{p}^{k}\right) \cap U\right]+V(T)+P\left[S_{k}+|E|-k\right] \\
=V(U)-V\left[\left\{M\left(\mathbf{p}^{k}\right) \cup E\left(\mathbf{p}^{k}\right)\right\} \cap U\right] \\
+V(T)+P\left[S_{k}+|E|-k\right] .
\end{gathered}
$$

Since

$$
\begin{aligned}
V\left[\left\{M\left(\mathbf{p}^{\mathrm{k}}\right) \cup E\left(\mathbf{p}^{k}\right)\right\} \cap U\right] & \leq P \sum\left\{p_{i}^{k}: \quad i \in\left\{M\left(\mathbf{p}^{k}\right) \cap E\left(p^{k}\right)\right\} \cup U\right\} \\
& \leq P \sum\left\{p_{i}^{k}: \quad i \in U\right\} \leq P(m-k),
\end{aligned}
$$

(8.26) follows from (8.28).

We finally turn to the proof of (8.27). The definition of $s$ [see (8.24)] implies $s \geq 0$. Since (8.25) implies $k>s$, we have $s<k \leq \mu$; thus $0 \leq s<\mu$, and so $P_{s}$ and $H_{s}$ are well-defined. Note that $s$ $<\mu \leq m$; also, the definition of $s$ gives $s>m-V(U) / P$. It follows that

$$
0<m-s<V(U) / P \text {. }
$$

Since $V_{i} / P<1$ for $i \epsilon U,(8.29)$ imp:ies that there exists a subset $W$ of $U$ such that

$$
m-s-1<V(W) / P<m-s .
$$

Since integer $m-s>0$, the first inequality implies that $W$ is nonempty; since $V_{i} / P$ for $i \in W$, it follows that

$$
V(W) / P<\min \{m-s,|W|\} .
$$

Thus there exist probabilities $\left\{p_{i}^{s}: \quad i \in W\right\}$, summing to the right-hand side of (8.31), for which $p_{i}^{s}>$ $V_{i} / P$ for $i \in W$. Since

$$
0 \leq \min \{m-s,|U|\}-\min \{m-s,|W|\} \leq|U-W|,
$$


there exist probabilities $\left\{p_{i}^{s}: i \epsilon U-W\right\}$ summing to the middle term in (8.32). Thus the probabilities $\left\{p_{i}^{s:} \quad i \in U\right\}$ sum to $\min \{m-s,|U|\}$, and so can be entered in (8.19) for the problem $P_{s}$, yielding

$$
\begin{aligned}
-H\left(\mathbf{p}^{s}\right) & =\sum\left\{V_{i} c_{i}^{*}: \quad i \epsilon E\left(\mathbf{p}^{s}\right) \cap U\right\}+V\left[L\left(\mathbf{p}^{s}\right) \cap U\right] \\
& +V(T)+P\left[S_{s}+|E|-s\right] .
\end{aligned}
$$

Thus

$$
\begin{aligned}
-H_{s} \leq-H\left(\mathbf{p}^{s}\right) & \leq V\left[\left\{E\left(\mathbf{p}^{s}\right) \cup L\left(\mathbf{p}^{s}\right)\right\} \cap U\right] \\
& +V(T)+P\left[S_{s}+|E|-s\right] \\
& =V(U)-V[M(\mathbf{p}) \cap U]+V(T)+P\left[S_{s}+|E|-s\right] .
\end{aligned}
$$

Since the choice of $\left\{p_{i}^{s:} \quad i \in W\right\}$ assures $W \subseteq M(\mathbf{p}) \cap U$, it follows that

$$
-H_{s} \leq V(U)-V(W)+V(T)+P\left[S_{s}+|E|-s\right],
$$

and this together with the first inequality in (8.30) imply (8.27), completing the proof.

\section{Index of Notation}

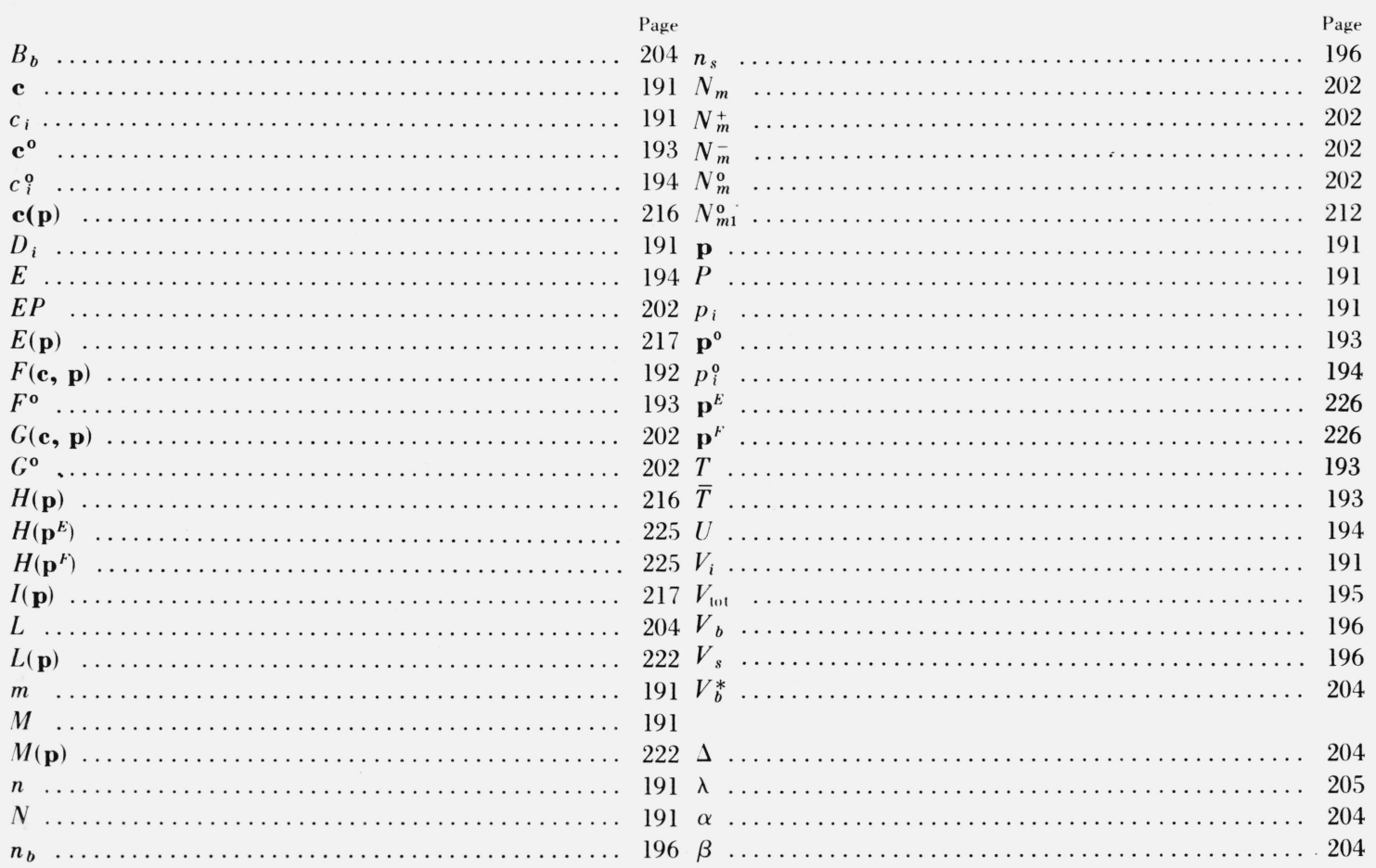

(Paper 80B2-437) 